\title{
Time-Splitting Procedures for the Numerical Solution of the 2D Advection-Diffusion Equation
}

\author{
A. R. Appadu ${ }^{1}$ and H. H. Gidey ${ }^{1,2}$ \\ ${ }^{1}$ Department of Mathematics and Applied Mathematics, University of Pretoria, Pretoria 0002, South Africa \\ ${ }^{2}$ African Institute for Mathematical Sciences (AIMS), 6 Road Melrose Road, Muizenberg 7945, Cape Town, South Africa \\ Correspondence should be addressed to A. R. Appadu; rao.appadu@up.ac.za
}

Received 5 June 2013; Accepted 12 August 2013

Academic Editor: Waqar Khan

Copyright (c) 2013 A. R. Appadu and H. H. Gidey. This is an open access article distributed under the Creative Commons Attribution License, which permits unrestricted use, distribution, and reproduction in any medium, provided the original work is properly cited.

\begin{abstract}
We perform a spectral analysis of the dispersive and dissipative properties of two time-splitting procedures, namely, locally onedimensional (LOD) Lax-Wendroff and LOD $(1,5)$ [9] for the numerical solution of the 2D advection-diffusion equation. We solve a 2D numerical experiment described by an advection-diffusion partial differential equation with specified initial and boundary conditions for which the exact solution is known. Some errors are computed, namely, the error rate with respect to the $L_{1}$ norm, dispersion and dissipation errors. Lastly, an optimization technique is implemented to find the optimal value of temporal step size that minimizes the dispersion error for both schemes when the spatial step is chosen as 0.025 , and this is validated by numerical experiments.
\end{abstract}

\section{Introduction}

The advection-diffusion equation is a parabolic partial differential equation combining the diffusion and advection (convection) equations, which describes physical phenomena where particles, energy, or other physical quantities are transferred inside a physical system due to two processes: diffusion and advection [1]. The numerical solution of advectiondiffusion equation plays an important role in many fields of science and engineering. These include the transport of air and groundwater pollutants, oil reservoir flow [2], heat transfer in draining film, flow through porous media, the dispersion of pollutants in rivers and streams, long range transport of pollutants in the atmosphere, thermal pollution in river systems, and dispersion of dissolved salts in groundwater [3].

The $3 \mathrm{D}$ advection-diffusion equation is given by

$$
\frac{\partial u}{\partial t}+\beta_{x} \frac{\partial u}{\partial x}+\beta_{y} \frac{\partial u}{\partial y}+\beta_{z} \frac{\partial u}{\partial z}=\alpha_{x} \frac{\partial^{2} u}{\partial x^{2}}+\alpha_{y} \frac{\partial^{2} u}{\partial y^{2}}+\alpha_{z} \frac{\partial^{2} u}{\partial z^{2}}
$$

where $\beta_{x}, \beta_{y}$, and $\beta_{z}$ are the velocity components of advection in the directions of $x, y$, and $z$, respectively, and $\alpha_{x}, \alpha_{y}$, and $\alpha_{z}$ are the coefficients of diffusivity in the $x$-, $y$-, and $z$ directions, respectively.

This study deals with the $2 \mathrm{D}$ advection-diffusion equation,

$$
\frac{\partial u}{\partial t}+\beta_{x} \frac{\partial u}{\partial x}+\beta_{y} \frac{\partial u}{\partial y}=\alpha_{x} \frac{\partial^{2} u}{\partial x^{2}}+\alpha_{y} \frac{\partial^{2} u}{\partial y^{2}},
$$

where $0<t \leq T$, in the domain $0 \leq x \leq 1,0 \leq y \leq 1$, with specified initial and boundary conditions.

Dehghan [3] proposed two time-splitting procedures for the solution of the two-dimensional transport equation. The time-splitting procedure used is the locally-one dimensional (LOD) in proceeding from one time step to the next step. LOD replaces the complicated multidimensional partial differential equations by a sequence of solutions of simpler onedimensional partial differential equations. The originality in this work is that we perform a spectral analysis of the dispersion and dissipation properties of the two schemes at some values of the temporal and spatial step sizes. 3D plots of the relative phase error per unit time step (RPE) and the modulus of the amplification factor (AFM) versus phase angles in $x$ - and $y$-directions are obtained. We then use optimization strategies to compute the optimal values of 
the temporal step size for the two schemes when the spatial step size is 0.025 . We then validate this result by performing a 2D numerical experiment with specified initial and boundary conditions. Lastly, we have the conclusion and references.

\section{Numerical Dispersion and Dissipation}

Dissipation reduces the amplitude of sinusoids in a Fourier series. This is caused by the presence of derivatives like $u_{x x}$ and $-u_{x x x x}$ in the modified equation [4]. On the other hand, the amplitude of sinusoids in a Fourier series is increased by antidissipation. Derivatives like $-u_{x x}$ and $u_{x x x x}$ in the modified equation are generally antidissipative. Dispersion affects the speed of sinusoids in a Fourier series causing phase lag or phase lead and is caused due to the presence of oddorder derivatives in the modified equation [4].

The modulus of the amplification factor (AFM) is a measure of the stability of a scheme and it is also used to measure the dissipative characteristics of the scheme. If the modulus of the amplification factor is equal to one, a disturbance neither grows nor damps [5]. If the modulus of the amplification factor is greater than one, then the scheme is unstable [6]; if it is less than one, damping occurs [7]. The partial differential equation given by (2) is dissipative in nature due to the terms $u_{x x}$ and $u_{y y}$.

The relative phase error (RPE) is a measure of the dispersive characteristics of a scheme. The relative phase error of a scheme approximating the $1 \mathrm{D}$ advection-diffusion equation is given by

$$
\mathrm{RPE}=-\frac{1}{c \omega} \arctan \left(\frac{\mathfrak{I}(\xi)}{\mathfrak{R}(\xi)}\right),
$$

where $c$ is the Courant number, $\omega$ is phase angle, $\xi$ is the amplification factor of the numerical scheme approximating the $1 \mathrm{D}$ advection-diffusion equation, and $\mathfrak{R}(\xi)$ and $\mathfrak{\Im}(\xi)$ are the real and imaginary parts of $\xi$, respectively [8].

We extend the work on the relative phase error in [8] for the case of the $2 \mathrm{D}$ advection-diffusion equation. The relative phase error for a numerical scheme approximating (2) is obtained on substituting $u$ by $\exp \left(I\left(\omega_{1} t-\theta_{1} x-\theta_{2} y\right)\right)$ [9], where $I=\sqrt{-1}, t$ is the time, $\theta_{1}$ and $\theta_{2}$ are wavenumbers in the directions of $x$ and $y$, respectively, and $\omega_{1}$ is the dispersion relation. Thus, we get

$$
\begin{gathered}
\frac{\partial u}{\partial t}=I \omega_{1} \exp \left(I\left(\omega_{1} t-\theta_{1} x-\theta_{2} y\right)\right), \\
\frac{\partial u}{\partial x}=-I \theta_{1} \exp \left(I\left(\omega_{1} t-\theta_{1} x-\theta_{2} y\right)\right), \\
\frac{\partial u}{\partial y}=-I \theta_{2} \exp \left(I\left(\omega_{1} t-\theta_{1} x-\theta_{2} y\right)\right), \\
\frac{\partial^{2} u}{\partial x^{2}}=-\left(\theta_{1}\right)^{2} \exp \left(I\left(\omega_{1} t-\theta_{1} x-\theta_{2} y\right)\right), \\
\frac{\partial^{2} u}{\partial y^{2}}=-\left(\theta_{2}\right)^{2} \exp \left(I\left(\omega_{1} t-\theta_{1} x-\theta_{2} y\right)\right)
\end{gathered}
$$

On substituting (4) into (2), we obtain

$$
\omega_{1}=\beta_{x} \theta_{1}+\beta_{y} \theta_{2}+\left(\alpha_{x}\left(\theta_{1}\right)^{2}\right) I+\left(\alpha_{y}\left(\theta_{2}\right)^{2}\right) I .
$$
obtain

The exact phase velocity is $\mathfrak{R}\left(\omega_{1}\right)$ /wavenumber and we

$$
\text { exact phase velocity }=\frac{\beta_{x} \theta_{1}+\beta_{y} \theta_{2}}{\text { wavenumber }} \text {. }
$$

The amplification factor can be written as $\xi=\xi_{1}+$ $I \xi_{2}$, where $\xi_{1}$ and $\xi_{2}$ are the real and imaginary parts of $\xi$, respectively. Also, we can express $\xi$ as $\xi=\exp (-b k)$, where $k$ is time step and $b$ is exponential growth rate [9]. Thus, we obtain

$$
b=\frac{1}{k} \log \left(\frac{\xi_{1}-I \xi_{2}}{\xi_{1}^{2}+\xi_{2}^{2}}\right) .
$$

The numerical phase velocity is calculated as $\mathfrak{\Im}(b)$ /wavenumber and we get

$$
\text { numerical phase velocity }=\frac{-(1 / k) \arctan \left(\xi_{2} / \xi_{1}\right)}{\text { wavenumber }} \text {. }
$$

The relative phase error is the ratio of the numerical phase velocity to the exact phase velocity [10]. It is calculated as

$$
\mathrm{RPE}=-\frac{1}{k\left(\beta_{x} \theta_{1}+\beta_{y} \theta_{2}\right)} \arctan \left(\frac{\xi_{2}}{\xi_{1}}\right) .
$$

The phase angles in the directions of $x$ and $y$ are given by $\omega_{x}=\Delta x \theta_{1}$ and $\omega_{y}=\Delta y \theta_{2}$, respectively. Hence, the relative phase error is

$$
\mathrm{RPE}=-\frac{1}{c_{x} \omega_{x}+c_{y} \omega_{y}} \arctan \left(\frac{\xi_{2}}{\xi_{1}}\right),
$$

where $c_{x}=\beta_{x} k / \Delta x$ and $c_{y}=\beta_{y} k / \Delta y$ are the Courant numbers in the directions of $x$ and $y$, respectively.

\section{Time-splitting Procedures and Numerical Experiments}

The domain we consider is $x, y \in[0,1]$. We divide the spatial interval $[0,1]$ along $x$ - and $y$-directions into $N$ and $M$ nodes, respectively, such that $(N-1) \Delta x=1$ and $(M-1) \Delta y=1$ and also divide the time interval $[0, T]$ into $P$ grid points such that $(P-1) \Delta t=T$. Then the grid points $\left(x_{i}, y_{j}, t_{n}\right)$ are defined by

$$
\begin{aligned}
& x_{i}=i \Delta x, \quad i=1,2, \ldots, N \\
& y_{j}=j \Delta y, \quad j=1,2, \ldots, M \\
& t_{n}=n \Delta t, \quad n=1,2, \ldots, P .
\end{aligned}
$$

For simplification we take $\Delta x=\Delta y=h$. Let $\Delta t=k$; then the parameters $h$ and $k$ represent the spatial and temporal grid spacing, respectively. We denote the approximated value of $u$ at the grid point $(i, j, n)$ by $u_{i, j}^{n}$. 
3.1. Time-Splitting Procedures. Since one-dimensional schemes are easier to use than two-dimensional schemes, (2) is split into the following two one-dimensional equations:

$$
\begin{aligned}
& \frac{1}{2} \frac{\partial u}{\partial t}+\beta_{x} \frac{\partial u}{\partial x}=\alpha_{x} \frac{\partial^{2} u}{\partial x^{2}} \\
& \frac{1}{2} \frac{\partial u}{\partial t}+\beta_{y} \frac{\partial u}{\partial y}=\alpha_{y} \frac{\partial^{2} u}{\partial y^{2}}
\end{aligned}
$$

Each of (12) and (13) can be solved over half of a time step to be used for the complete $2 \mathrm{D}$ advection-diffusion equation, using the procedures developed for the $1 \mathrm{D}$ advectiondiffusion equation.

Some work on time-splitting procedures can be found in $[3,11]$. In this paper, we refer to [3] on how a $2 \mathrm{D}$ advectiondiffusion equation is converted into two $1 \mathrm{D}$ advectiondiffusion equations using the locally one-dimensional (LOD) time-splitting procedure. Solving (12) and (13) in each half time step is equivalent to solving the following equations over a full-time step:

$$
\begin{aligned}
& \frac{\partial u}{\partial t}+\beta_{x} \frac{\partial u}{\partial x}=\alpha_{x} \frac{\partial^{2} u}{\partial x^{2}} \\
& \frac{\partial u}{\partial t}+\beta_{y} \frac{\partial u}{\partial y}=\alpha_{y} \frac{\partial^{2} u}{\partial y^{2}}
\end{aligned}
$$

Then we can use the schemes used for solving the 1D advection-diffusion equation to solve (14) and (15).

3.2. Numerical Experiments. In [12], different explicit and implicit finite differences schemes are used to solve the $1 \mathrm{D}$ advection-diffusion equation

$$
\frac{\partial u}{\partial t}+\frac{\partial u}{\partial x}=0.01 \frac{\partial^{2} u}{\partial x^{2}}
$$

Three values of the cell Reynolds number, namely, $R_{\Delta}=2,4$, and 8 , are used for the numerical experiments. In [3], the time-splitting procedure is used to solve the two-dimensional transport equation in the region $0 \leq x \leq 1,0 \leq y \leq 1$, with

$$
\alpha_{x}=\alpha_{y}=\alpha=0.01, \quad \beta_{x}=\beta_{y}=\beta=0.8
$$

and with the following boundary and initial conditions:

$$
\begin{aligned}
& g_{0}(x, t)=\frac{1}{4 t+1} \exp \left[-\frac{(x-0.8 t-0.5)^{2}}{0.01(4 t+1)}-\frac{(0.8 t+0.5)^{2}}{0.01(4 t+1)}\right], \\
& g_{1}(x, t)=\frac{1}{4 t+1} \exp \left[-\frac{(x-0.8 t-0.5)^{2}}{0.01(4 t+1)}-\frac{(0.5-0.8 t)^{2}}{0.01(4 t+1)}\right],
\end{aligned}
$$

$$
h_{0}(y, t)=\frac{1}{4 t+1} \exp \left[-\frac{(0.8 t+0.5)^{2}}{0.01(4 t+1)}-\frac{(y-0.8 t-0.5)^{2}}{0.01(4 t+1)}\right] \text {, }
$$

$$
h_{1}(y, t)=\frac{1}{4 t+1} \exp \left[-\frac{(0.5-0.8 t)^{2}}{0.01(4 t+1)}-\frac{(y-0.8 t-0.5)^{2}}{0.01(4 t+1)}\right] \text {, }
$$

$$
f(x, y)=\exp \left[-\frac{(x-0.5)^{2}}{0.01}-\frac{(y-0.5)^{2}}{0.01}\right],
$$

for which the exact solution is

$$
\begin{array}{r}
u(x, y, t)=\frac{1}{4 t+1} \exp \left[-\frac{(x-0.8 t-0.5)^{2}}{0.01(4 t+1)}\right. \\
\left.-\frac{(y-0.8 t-0.5)^{2}}{0.01(4 t+1)}\right] .
\end{array}
$$

We consider two time-splitting procedures LOD LaxWendroff and $\operatorname{LOD}(1,5)$ to solve $(2)$ with $\alpha_{x}=\alpha_{y}=\alpha=0.01$ and $\beta_{x}=\beta_{y}=\beta=0.8$, subject to the boundary conditions (18) $-(22)$ at time $T=0.3$.

We consider two values of $R_{\Delta} ; R_{\Delta}=2$ and $R_{\Delta}=4$. Since $c=0.8 \mathrm{k} / \mathrm{h}$ and $s=0.01 \mathrm{k} / \mathrm{h}^{2}$, we have $R_{\Delta}=80 \mathrm{~h}$. Thus for $R_{\Delta}=2$ and 4 , we have $h=0.025$ and $h=0.05$, respectively. When $h=0.025$, we have $c=32 k$ and $s=16 k$ and for $h=$ 0.05 we have $c=16 k$ and $s=4 k$.

3.3. Quantification of Errors from Numerical Results. In this subsection, we describe how errors from numerical results can be quantified into dispersion and dissipation by a technique devised by Takacs [13].

The total mean square error in the $1 \mathrm{D}$ case [13] is calculated as

$$
E_{\mathrm{TMS}}=\frac{1}{N} \sum_{i=1}^{N}\left(u_{i}-v_{i}\right)^{2} \text {, }
$$

where $u_{i}$ is the exact solution, $v_{i}$ is the numerical solution at a grid point $i$, and $N$ is the number of grid points. The total mean square error can be expressed as

$$
\begin{aligned}
\frac{1}{N} \sum_{i=1}^{N}\left(u_{i}-v_{i}\right)^{2}= & \frac{1}{N} \sum_{i=1}^{N}\left(u_{i}-\bar{u}\right)^{2}+\frac{1}{N} \sum_{i=1}^{N}\left(v_{i}-\bar{v}\right)^{2} \\
& +\frac{2}{N} \sum_{i=1}^{N} u_{i} \bar{u}+\frac{2}{N} \sum_{i=1}^{N} v_{i} \bar{v}-\frac{1}{N} \sum_{i=1}^{N}(\bar{u})^{2} \\
& -\frac{1}{N} \sum_{i=1}^{N}(\bar{v})^{2}-\frac{2}{N} \sum_{i=1}^{N} u_{i} v_{i} .
\end{aligned}
$$

The right hand side of (25) can be rewritten as

$$
\sigma^{2}(u)+\sigma^{2}(v)+2(\bar{u})^{2}+2(\bar{v})^{2}-(\bar{u})^{2}-(\bar{v})^{2}-\frac{2}{N} \sum_{i=1}^{N} u_{i} v_{i},
$$


where $\sigma^{2}(u)$ and $\sigma^{2}(v)$ denote the variance of $u$ and $v$, respectively, and $\bar{u}$ and $\bar{v}$ denote the mean values of $u$ and $v$, respectively. Then we have

$$
\begin{aligned}
E_{\mathrm{TMS}}= & \sigma^{2}(u)+\sigma^{2}(v)+\left((\bar{u})^{2}-2 \bar{u} \bar{v}+(\bar{v})^{2}\right) \\
& +\left(2 \bar{u} \bar{v}-\frac{2}{N} \sum_{i=1}^{N} u_{i} v_{i}\right) \\
= & \sigma^{2}(u)+\sigma^{2}(v)+(\bar{u}-\bar{v})^{2}-2\left(\frac{1}{N} \sum_{i=1}^{N} u_{i} v_{i}-\bar{u} \bar{v}\right) .
\end{aligned}
$$

Therefore,

$$
\frac{1}{N} \sum_{i=1}^{N}\left(u_{i}-v_{i}\right)^{2}=\sigma^{2}(u)+\sigma^{2}(v)+(\bar{u}-\bar{v})^{2}-2 \operatorname{Cov}(u, v)
$$

where $\operatorname{Cov}(u, v)=(1 / N)\left(\sum_{i=1}^{N} u_{i} v_{i}-\bar{u} \bar{v}\right)$.

The total mean square error can be expressed as

$$
\begin{aligned}
\frac{1}{N} \sum_{i=1}^{N}\left(u_{i}-v_{i}\right)^{2}= & (\sigma(u)-\sigma(v))^{2} \\
& +(\bar{u}-\bar{v})^{2}+2(1-\rho) \sigma(u) \sigma(v),
\end{aligned}
$$

where $\rho=\operatorname{Cov}(u, v) /(\sigma(u) \sigma(v))$ is the coefficient of correlation.

The term $2(1-\rho) \sigma(u) \sigma(v)$ measures the dispersion error and the term $(\sigma(u)-\sigma(v))^{2}+(\bar{u}-\bar{v})^{2}$ measures the dissipation error.

We extend the work on quantification of errors in $[14,15]$ for the $2 \mathrm{D}$ case. The total mean square error for the $2 \mathrm{D}$ case is calculated as

$$
E_{\mathrm{TMS}}=\frac{1}{N M} \sum_{j=1}^{M} \sum_{i=1}^{N}\left(u_{i, j}-v_{i, j}\right)^{2}
$$

where $u_{i, j}$ and $v_{i, j}$ are the exact and numerical solutions at a grid point $(i, j)$, respectively, and

$$
\begin{aligned}
& \frac{1}{N M} \sum_{j=1}^{M} \sum_{i=1}^{N}\left(u_{i, j}-v_{i, j}\right)^{2} \\
& \quad=\frac{1}{N M} \sum_{j=1}^{M} \sum_{i=1}^{N}\left(u_{i, j}^{2}-2 u_{i, j} v_{i, j}+v_{i, j}^{2}\right) \\
& =\frac{1}{N M}\left(\sum_{j=1}^{M} \sum_{i=1}^{N} u_{i, j}^{2}+\sum_{j=1}^{M} \sum_{i=1}^{N} v_{i, j}^{2}-2 \sum_{j=1}^{M} \sum_{i=1}^{N} u_{i, j} v_{i, j}\right) .
\end{aligned}
$$

Since

$$
\begin{aligned}
& \sum_{j=1}^{M} \sum_{i=1}^{N}\left(u_{i, j}-\bar{u}\right)^{2}=\sum_{j=1}^{M} \sum_{i=1}^{N}\left(u_{i, j}^{2}-2 u_{i, j} \bar{u}+\bar{u}^{2}\right), \\
& \sum_{j=1}^{M} \sum_{i=1}^{N}\left(v_{i, j}-\bar{v}\right)^{2}=\sum_{j=1}^{M} \sum_{i=1}^{N}\left(v_{i, j}^{2}-2 v_{i, j} \bar{v}+\bar{v}^{2}\right),
\end{aligned}
$$

we have

$$
\begin{aligned}
E_{\mathrm{TMS}}= & \frac{1}{N M}\left[\sum_{j=1}^{M} \sum_{i=1}^{N}\left(u_{i, j}-\bar{u}\right)^{2}+\sum_{j=1}^{M} \sum_{i=1}^{N}\left(v_{i, j}-\bar{v}\right)^{2}\right. \\
& +2 \sum_{j=1}^{M} \sum_{i=1}^{N} u_{i, j} \bar{u}+2 \sum_{j=1}^{M} \sum_{i=1}^{N} v_{i, j} \bar{v} \\
& \left.-2 \sum_{j=1}^{M} \sum_{i=1}^{N} u_{i, j} v_{i, j}-\sum_{j=1}^{M} \sum_{i=1}^{N} \bar{u}^{2}-\sum_{j=1}^{M} \sum_{i=1}^{N} \bar{v}^{2}\right] \\
= & \sigma^{2}(u)+\sigma^{2}(v)+2 \bar{u}^{2}+2 \bar{v}^{2} \\
& \frac{2}{N M} \sum_{j=1}^{M} \sum_{i=1}^{N} u_{i, j} v_{i, j}-\bar{u}^{2}-\bar{v}^{2} \\
= & \sigma^{2}(u)+\sigma^{2}(v)+\left(\bar{u}^{2}-2 \bar{u} \bar{v}+\bar{v}^{2}\right) \\
& +2 \bar{u} \bar{v}-\frac{2}{N M} \sum_{j=1}^{M} \sum_{i=1}^{N} u_{i, j} v_{i, j} \\
= & \sigma^{2}(u)+\sigma^{2}(v)+(\bar{u}-\bar{v})^{2} \\
& \left.\frac{1}{N M} \sum_{j=1}^{M} \sum_{i=1}^{N} u_{i, j} v_{i, j}-\bar{u} \bar{v}\right]
\end{aligned}
$$

Hence,

$$
E_{\mathrm{TMS}}=(\sigma(u)-\sigma(v))^{2}+(\bar{u}-\bar{v})^{2}+2(1-\rho) \sigma(u) \sigma(v),
$$

where $\rho=\operatorname{Cov}(u, v) / \sigma(u) \sigma(v)$. The dissipation error is $(\sigma(u)-\sigma(v))^{2}+(\bar{u}-\bar{v})^{2}$ and the dispersion error is $2(1-$ $\rho) \sigma(u) \sigma(v)$.

The error rate with respect to $L_{1}$ norm for $x, y \in[0,1]$ is calculated as

$$
E_{\text {num }}=\frac{1}{(N-1)(M-1)} \sum_{j=1}^{M} \sum_{i=1}^{N}\left|u_{i, j}-v_{i, j}\right| .
$$

\section{Construction of the LOD Lax-Wendroff Procedure}

We use the following approximations in the first time step of the LOD procedure [3]:

$$
\left.\frac{\partial u}{\partial t}\right|_{i, j} ^{n} \simeq \frac{u_{i, j}^{n+(1 / 2)}-u_{i, j}^{n}}{\Delta t}
$$




$$
\begin{gathered}
\left.\frac{\partial u}{\partial x}\right|_{i, j} ^{n} \simeq \frac{\left(1-\gamma_{x}\right)\left(u_{i, j}^{n}-u_{i-1, j}^{n}\right)+\gamma_{x}\left(u_{i+1, j}^{n}-u_{i, j}^{n}\right)}{\Delta x}, \\
\left.\frac{\partial^{2} u}{\partial x^{2}}\right|_{i, j} ^{n} \simeq \frac{u_{i+1, j}^{n}-2 u_{i, j}^{n}-u_{i-1, j}^{n}}{(\Delta x)^{2}},
\end{gathered}
$$

where $\gamma_{x}$ is the spatial weighting factor in the direction of $x$. The following approximations are used for the second step of the LOD procedure:

$$
\begin{gathered}
\left.\frac{\partial u}{\partial t}\right|_{i, j} ^{n+(1 / 2)} \simeq \frac{u_{i, j}^{n+1}-u_{i, j}^{n+(1 / 2)}}{\Delta t}, \\
\left.\frac{\partial u}{\partial y}\right|_{i, j} ^{n+(1 / 2)} \\
\simeq \frac{\left(1-\gamma_{y}\right)\left(u_{i, j}^{n+(1 / 2)}-u_{i, j-1}^{n+(1 / 2)}\right)+\gamma_{y}\left(u_{i, j+1}^{n+(1 / 2)}-u_{i, j}^{n+(1 / 2)}\right)}{\Delta y}, \\
\left.\frac{\partial^{2} u}{\partial y^{2}}\right|_{i, j} ^{n+(1 / 2)} \simeq \frac{u_{i, j+1}^{n+(1 / 2)}-2 u_{i, j}^{n+(1 / 2)}-u_{i, j-1}^{n+(1 / 2)}}{(\Delta y)^{2}},
\end{gathered}
$$

where $\gamma_{y}$ is the spatial weighting factor in the direction of $y$. By using the following relationships

$$
\begin{array}{lll}
c_{x}=\frac{\beta_{x} k}{h}, & s_{x}=\frac{\alpha_{x} k}{h^{2}}, & \gamma_{x}=\frac{1-c_{x}}{2}, \\
c_{y}=\frac{\beta_{y} k}{h}, & s_{y}=\frac{\alpha_{y} k}{h^{2}}, & \gamma_{y}=\frac{1-c_{y}}{2},
\end{array}
$$

the finite difference formula for the first half time step of the LOD Lax-Wendroff procedure is given by

$$
\begin{aligned}
u_{i, j}^{n+(1 / 2)}= & \frac{1}{2}\left(2 s_{x}+c_{x}+c_{x}^{2}\right) u_{i-1, j}^{n}+\left(1-2 s_{x}-c_{x}^{2}\right) u_{i, j}^{n} \\
& +\frac{1}{2}\left(2 s_{x}-c_{x}+c_{x}^{2}\right) u_{i+1, j}^{n} .
\end{aligned}
$$

At the second half time step the finite difference is given by

$$
\begin{aligned}
u_{i, j}^{n+1}= & \frac{1}{2}\left(2 s_{y}+c_{y}+c_{y}^{2}\right) u_{i, j-1}^{n+(1 / 2)}+\left(1-2 s_{y}-c_{y}^{2}\right) u_{i, j}^{n+(1 / 2)} \\
& +\frac{1}{2}\left(2 s_{y}-c_{y}+c_{y}^{2}\right) u_{i, j+1}^{n+(1 / 2)}
\end{aligned}
$$

where

$$
\begin{aligned}
u_{i, j-1}^{n+(1 / 2)}= & \frac{1}{2}\left(2 s_{x}+c_{x}+c_{x}^{2}\right) u_{i-1, j-1}^{n}+\left(1-2 s_{x}-c_{x}^{2}\right) u_{i, j-1}^{n} \\
& +\frac{1}{2}\left(2 s_{x}-c_{x}+c_{x}^{2}\right) u_{i+1, j-1}^{n}, \\
u_{i, j+1}^{n+(1 / 2)}= & \frac{1}{2}\left(2 s_{x}+c_{x}+c_{x}^{2}\right) u_{i-1, j+1}^{n}+\left(1-2 s_{x}-c_{x}^{2}\right) u_{i, j+1}^{n} \\
& +\frac{1}{2}\left(2 s_{x}-c_{x}+c_{x}^{2}\right) u_{i+1, j+1}^{n} .
\end{aligned}
$$

We obtain a single expression for $u_{i, j}^{n+1}$ in a complete time step as follows:

$$
\begin{aligned}
u_{i, j}^{n+1}= & \frac{1}{4}\left(2 s_{y}+c_{y}+c_{y}^{2}\right)\left(2 s_{x}+c_{x}+c_{x}^{2}\right) u_{i-1, j-1}^{n} \\
& +\frac{1}{2}\left(2 s_{y}+c_{y}+c_{y}^{2}\right)\left(1-2 s_{x}-c_{x}^{2}\right) u_{i, j-1}^{n} \\
& +\frac{1}{4}\left(2 s_{y}+c_{y}+c_{y}^{2}\right)\left(2 s_{x}-c_{x}+c_{x}^{2}\right) u_{i+1, j-1}^{n} \\
& +\frac{1}{2}\left(1-2 s_{y}-c_{y}^{2}\right)\left(2 s_{x}+c_{x}+c_{x}^{2}\right) u_{i-1, j}^{n} \\
& +\left(1-2 s_{y}-c_{y}^{2}\right)\left(1-2 s_{x}-c_{x}^{2}\right) u_{i, j}^{n} \\
& +\frac{1}{2}\left(1-2 s_{y}-c_{y}^{2}\right)\left(2 s_{x}-c_{x}+c_{x}^{2}\right) u_{i+1, j}^{n} \\
& +\frac{1}{4}\left(2 s_{y}-c_{y}+c_{y}^{2}\right)\left(2 s_{x}+c_{x}+c_{x}^{2}\right) u_{i-1, j+1}^{n} \\
& +\frac{1}{2}\left(2 s_{y}-c_{y}+c_{y}^{2}\right)\left(1-2 s_{x}-c_{x}^{2}\right) u_{i, j+1}^{n} \\
& +\frac{1}{4}\left(2 s_{y}-c_{y}+c_{y}^{2}\right)\left(2 s_{x}-c_{x}+c_{x}^{2}\right) u_{i+1, j+1}^{n}
\end{aligned}
$$

To find the modified equation of the scheme, we first find Taylor's expansion of each term in (42) about $u_{i, j}^{n}$. The Taylor series expansion of $u_{i, j}^{n+1}$ is given by

$$
u_{i, j}^{n+1}=u+k u_{t}+\frac{k^{2}}{2} u_{t t}+\frac{k^{3}}{6} u_{t t t}+\frac{k^{4}}{4 !} u_{t t t t}+\cdots
$$

The Taylor series expansions for some grid points about $u_{i, j}^{n}$ are given as follows:

$$
\begin{aligned}
u_{i-1, j-1}^{n}= & u-h u_{x}-h u_{y}+\frac{h^{2}}{2}\left[u_{x x}+2 u_{x y}+u_{y y}\right] \\
& -\frac{h^{3}}{6}\left[u_{x x x}+3 u_{x x y}+3 u_{x y y}+u_{y y y}\right] \\
+ & \frac{h^{4}}{4 !}\left[u_{x x x x}+4 u_{x x x y}+6 u_{x x y y}\right. \\
& \left.+4 u_{x y y y}+u_{y y y y}\right]+\cdots,
\end{aligned}
$$




$$
\begin{aligned}
& u_{i-1, j}^{n}=u-h u_{x}+\frac{h^{2}}{2} u_{x x}-\frac{h^{3}}{6} u_{x x x}+\frac{h^{4}}{4 !} u_{x x x x}+\cdots \\
& u_{i, j-1}^{n}=u-h u_{y}+\frac{h^{2}}{2} u_{y y}-\frac{h^{3}}{6} u_{y y y}+\frac{h^{4}}{4 !} u_{y y y y}+\cdots, \\
& u_{i-1, j+1}^{n}=u-h u_{x}+h u_{y}+\frac{h^{2}}{2}\left[u_{x x}-2 u_{x y}+u_{y y}\right] \\
& -\frac{h^{3}}{6}\left[u_{x x x}-3 u_{x x y}+3 u_{x y y}-u_{y y y}\right] \\
& +\frac{h^{4}}{4 !}\left[u_{x x x x}-4 u_{x x x y}+6 u_{x x y y}\right. \\
& \left.-4 u_{x y y y}+u_{y y y y}\right]+\cdots, \\
& u_{i+1, j-1}^{n}=u+h u_{x}-h u_{y}+\frac{h^{2}}{2}\left[u_{x x}-2 u_{x y}+u_{y y}\right] \\
& -\frac{h^{3}}{6}\left[-u_{x x x}+3 u_{x x y}-3 u_{x y y}+u_{y y y}\right] \\
& +\frac{h^{4}}{4 !}\left[u_{x x x x}-4 u_{x x x y}\right. \\
& \left.+6 u_{x x y y}-4 u_{x y y y}+u_{y y y y}\right]+\cdots, \\
& u_{i+1, j}^{n}=u+h u_{x}+\frac{h^{2}}{2} u_{x x}+\frac{h^{3}}{6} u_{x x x}+\frac{h^{4}}{4 !} u_{x x x x}+\cdots \\
& u_{i, j+1}^{n}=u+h u_{y}+\frac{h^{2}}{2} u_{y y}+\frac{h^{3}}{6} u_{y y y}+\frac{h^{4}}{4 !} u_{y y y y}+\cdots, \\
& u_{i+1, j+1}^{n}=u+h u_{x}+h u_{y}+\frac{h^{2}}{2}\left[u_{x x}+2 u_{x y}+u_{y y}\right] \\
& +\frac{h^{3}}{6}\left[u_{x x x}+3 u_{x x y}+3 u_{x y y}+u_{y y y}\right] \\
& +\frac{h^{4}}{4 !}\left[u_{x x x x}+4 u_{x x x y}+6 u_{x x y y}\right. \\
& \left.+4 u_{x y y y}+u_{y y y y}\right]+\cdots .
\end{aligned}
$$

Using (2), we convert the temporal derivatives $u_{t t}, u_{t t t}$, and $u_{t t t t}$ into spatial derivatives. We thus have

$$
\begin{aligned}
u_{t t}= & \alpha_{x}^{2} u_{x x x x}+\alpha_{y}^{2} u_{y y y y}+\alpha_{x} \alpha_{y}\left[u_{x x y y}+u_{y y x x}\right] \\
& -2 \alpha_{x} \beta_{x} u_{x x x}-2 \alpha_{y} \beta_{y} u_{y y y}-\alpha_{x} \beta_{y}\left[u_{x x y}+u_{y x x}\right] \\
& -\alpha_{y} \beta_{x}\left[u_{x y y}+u_{y y x}\right]+\beta_{x}^{2} u_{x x}+\beta_{y}^{2} u_{y y} \\
& +\beta_{x} \beta_{y}\left[u_{x y}+u_{y x}\right]+\cdots \\
u_{t t t}= & 3 \alpha_{x} \beta_{x}^{2} u_{x x x x}+3 \alpha_{y} \beta_{y}^{2} u_{y y y y} \\
& +\alpha_{x} \beta_{y}^{2}\left[u_{y y x x}+u_{y x x y}+u_{x x y y}\right] \\
& +\alpha_{y} \beta_{x}^{2}\left[u_{x x y y}+u_{y y x x}+u_{x y y x}\right]
\end{aligned}
$$

$$
\begin{aligned}
+ & \alpha_{y} \beta_{x} \beta_{y}\left[2 u_{y y y x}+u_{y y x y}+2 u_{x y y y}+2 u_{y x y y}\right] \\
+ & \alpha_{x} \beta_{x} \beta_{y}\left[2 u_{y x x x}+2 u_{x x x y}+u_{x x y x}+2 u_{x y x x}\right] \\
& -\beta_{x}^{3} u_{x x x}-\beta_{x} \beta_{y}^{2}\left[u_{y y x}+u_{y x y}+u_{x y y}\right] \\
& -\beta_{y}^{3} u_{y y y}-\beta_{x}^{2} \beta_{y}\left[u_{x x y}+u_{x y x}+u_{y x x}\right]+\cdots \\
u_{t t t t} & \beta_{x}\left[\beta_{x}^{3} u_{x x x x}+\beta_{x}^{2} \beta_{y}\left[u_{x x y x}+u_{x y x x}+u_{y x x x}\right]\right. \\
& \left.+\beta_{x} \beta_{y}^{2}\left[u_{y y x x}+u_{y x y x}+{ }_{x y y x}\right]+\beta_{y}^{3} u_{y y y x}\right] \\
& +\beta_{y}\left[\beta_{y}^{3} u_{y y y y}+\beta_{y}^{2} \beta_{x}\left[u_{y y x y}+u_{y x y y}+u_{x y y y}\right]\right. \\
& \left.+\beta_{y} \beta_{x}^{2}\left[u_{x x y y}+u_{x y x y}+u_{y x x y}\right]+\beta_{x}^{3} u_{x x x y}\right]+\cdots
\end{aligned}
$$

Then on substituting the Taylor series expansions of each term of the difference scheme we get the following modified equation for the LOD Lax-Wendroff

$$
\begin{aligned}
u_{t}+ & \beta_{x} u_{x}+\beta_{y} u_{y} \\
= & \alpha_{x} u_{x x}+\alpha_{y} u_{y y}-\frac{1}{6} \beta_{x} h^{2}\left(1-c_{x}^{2}-6 s_{x}\right) u_{x x x} \\
& -\frac{1}{6} \beta_{y} h^{2}\left(1-c_{y}^{2}-6 s_{y}\right) u_{y y y} \\
& -\frac{h^{3}}{24}\left[-2 s_{x}-c_{x}^{2}+12 s_{x} c_{x}^{2}+12 s_{x}^{2}+c_{x}^{4}\right] u_{x x x x} \\
& -\frac{h^{3}}{24}\left[-2 s_{y}-c_{y}^{2}+12 s_{y} c_{y}^{2}+12 s_{y}^{2}+c_{y}^{4}\right] u_{y y y y}+\cdots
\end{aligned}
$$

The scheme is second order accurate in space and the leading error terms are dispersive in nature (presence of odd-order derivatives $u_{x x x}$ and $\left.u_{y y y}\right)$. As the time and spatial increments go to zero, the modified equation (46) reduces to its original equation, that is, (2). Hence LOD Lax-Wendroff is consistent.

We now study the spectral analysis of the dispersive and dissipative properties of the scheme for the case $c_{x}=c_{y}=c$ and $s_{x}=s_{y}=s$. To obtain the amplification factor we use the Von Neumann stability analysis by substituting $u_{i, j}^{n}$ by $\xi^{n} \exp \left(I\left(i \omega_{x}+j \omega_{y}\right)\right)$ in (42), where $I=\sqrt{-1}$. We thus have

$$
\begin{aligned}
\xi= & \frac{1}{4}\left(2 s_{y}+c_{y}+c_{y}^{2}\right)\left(2 s_{x}+c_{x}+c_{x}^{2}\right) \exp \left(-I\left(\omega_{x}+\omega_{y}\right)\right) \\
& +\frac{1}{2}\left(2 s_{y}+c_{y}+c_{y}^{2}\right)\left(1-2 s_{x}-c_{x}^{2}\right) \exp \left(-I \omega_{y}\right) \\
& +\frac{1}{4}\left(2 s_{y}+c_{y}+c_{y}^{2}\right)\left(2 s_{x}-c_{x}+c_{x}^{2}\right) \exp \left(I\left(\omega_{x}-\omega_{y}\right)\right) \\
& +\frac{1}{2}\left(1-2 s_{y}-c_{y}^{2}\right)\left(2 s_{x}+c_{x}+c_{x}^{2}\right) \exp \left(-I \omega_{x}\right) \\
& +\left(1-2 s_{y}-c_{y}^{2}\right)\left(1-2 s_{x}-c_{x}^{2}\right)
\end{aligned}
$$




$$
\begin{aligned}
& +\frac{1}{2}\left(1-2 s_{y}-c_{y}^{2}\right)\left(2 s_{x}-c_{x}+c_{x}^{2}\right) \exp \left(I \omega_{x}\right) \\
& +\frac{1}{4}\left(2 s_{y}-c_{y}+c_{y}^{2}\right)\left(2 s_{x}+c_{x}+c_{x}^{2}\right) \exp \left(-I\left(\omega_{x}-\omega_{y}\right)\right) \\
& +\frac{1}{2}\left(2 s_{y}-c_{y}+c_{y}^{2}\right)\left(1-2 s_{x}-c_{x}^{2}\right) \exp \left(I \omega_{y}\right) \\
& +\frac{1}{4}\left(2 s_{y}-c_{y}+c_{y}^{2}\right)\left(2 s_{x}-c_{x}+c_{x}^{2}\right) \exp \left(I\left(\omega_{x}+\omega_{y}\right)\right)
\end{aligned}
$$

The real and imaginary parts of the amplification factor are given by

$$
\begin{aligned}
\operatorname{Real}(\xi)= & 1+4 s c^{2} \cos \left(\omega_{x}\right) \cos \left(\omega_{y}\right)+c^{4} \cos \left(\omega_{x}\right) \cos \left(\omega_{y}\right) \\
& +4 s^{2} \cos \left(\omega_{x}\right) \cos \left(\omega_{y}\right)-4 s c^{2} \cos \left(\omega_{y}\right) \\
& -c^{2} \sin \left(\omega_{x}\right) \sin \left(\omega_{y}\right)+4 s^{2}+4 s c^{2}-4 s c^{2} \cos \left(\omega_{x}\right) \\
& +c^{2} \cos \left(\omega_{x}\right)-c^{4} \cos \left(\omega_{x}\right)+4 s \cos \left(\omega_{x}\right) \\
& +2 s \cos \left(\omega_{y}\right)+2 s \cos \left(\omega_{x}\right)-4 s^{2} \cos \left(\omega_{y}\right) \\
& -4 s^{2} \cos \left(\omega_{x}\right)-2 c^{2}+c^{2} \cos \left(\omega_{y}\right) \\
& -c^{4} \cos \left(\omega_{y}\right)+c^{4}-4 s, \\
\operatorname{Imag}(\xi)= & -c^{3} \cos \left(\omega_{x}\right) \sin \left(\omega_{y}\right)+2 s c \sin \left(\omega_{x}\right) \\
& +2 s c \sin \left(\omega_{y}\right)-2 s c \cos \left(\omega_{x}\right) \sin \left(\omega_{y}\right) \\
& -2 s c \sin \left(\omega_{x}\right) \cos \left(\omega_{y}\right)-c \sin \left(\omega_{x}\right)+c^{3} \sin \left(\omega_{x}\right) \\
& -c \sin \left(\omega_{y}\right)+c^{3} \sin \left(\omega_{y}\right)-c^{3} \sin \left(\omega_{x}\right) \cos \left(\omega_{y}\right),
\end{aligned}
$$

respectively. The modulus of the amplification factor, AFM, is obtained as

$$
\mathrm{AFM}=\sqrt{(\mathfrak{R}(\xi))^{2}+(\mathfrak{I}(\xi))^{2}}
$$

We find the region of stability using the approach of Hindmarsh et al. [16] and Sousa [17]. We consider the case when $\omega_{x}=\omega_{y}=\pi$ and $\omega_{x} \rightarrow 0$ and $\omega_{y} \rightarrow 0$. When $\omega_{x}=\omega_{y}=\pi$, (49) gives

$$
|\xi|=\sqrt{1+16 s c^{2}-4 c^{2}+4 c^{4}+16 s^{2}-8 s} .
$$

From the Von Neumann stability analysis, the scheme is stable if and only if $|\xi| \leq 1$. Thus, we get

$$
1+16 s c^{2}-4 c^{2}+4 c^{4}+16 s^{2}-8 s \leq 1 .
$$

On simplification, we get

$$
\left|s+\frac{2 c^{2}-1}{4}\right| \leq \frac{1}{4},
$$

which reduces to

$$
s \leq \frac{1-c^{2}}{2}
$$

When $\omega_{x} \rightarrow 0$ and $\omega_{y} \rightarrow 0$, we use the following approximations:

$$
\begin{array}{cl}
\cos \left(\omega_{x}\right) \simeq 1-\frac{\omega_{x}^{2}}{2}, & \cos \left(\omega_{y}\right) \simeq 1-\frac{\omega_{y}^{2}}{2}, \\
\sin \left(\omega_{x}\right) \simeq \omega_{x}, \quad \sin \left(\omega_{y}\right) \simeq \omega_{y} .
\end{array}
$$

We consider (49) and use the approximations in (54) to obtain

$$
|\xi|^{2} \simeq 1-2 s\left(\omega_{x}^{2}+\omega_{y}^{2}\right) .
$$

Thus LOD Lax-Wendroff is stable if $-2 s \leq 0$. Therefore, we have

$$
s \geq 0
$$

On combining (53) and (56), we obtain the region of stability for the LOD Lax-Wendroff procedure as

$$
0 \leq s \leq \frac{1-c^{2}}{2}
$$

We choose $\alpha=0.01$ and $\beta=0.8$ [3]. For $h=0.025$ from (57), we have

$$
0 \leq \frac{0.01 k}{(0.025)^{2}} \leq \frac{1-(0.8 k / 0.025)^{2}}{2} .
$$

On solving for $k$, we get

$$
0 \leq k \leq 0.0193
$$

Therefore, for $h=0.025$, the stability region for the LOD Lax-Wendroff procedure is $0 \leq k \leq 0.0193$. We choose $k$ such that $0.3 / k$ is an integer as for our numerical experiments, $T=0.3$. Then $0.3 / k$ gives the number of time intervals. We choose $k=0.005,0.01,3 / 160$, and $1 / 60$.

For $h=0.05$, we have $c=16 k$ and $s=4 k$. When $\omega_{x}=\pi$ and $\omega_{y}=\pi$, using (51) we get

$$
0 \leq 1-32 k+1638 k^{3}-768 k^{2}+262144 k^{4} \leq 1,
$$

which gives

$$
0 \leq k \leq 0.0487985
$$

Therefore, for $h=0.05$, LOD Lax-Wendroff is stable if $0 \leq k \leq$ 0.0487985 . We then choose some values of $k \in(0,0.048)$ for the numerical experiments. for our numerical experiments.

$3 \mathrm{D}$ plots of the modulus of the amplification factor versus phase angles in $x$-and $y$-directions for some different values of $h$ and $k$ are depicted in Figures 1 and 2. 2D plots of the modulus of the amplification factor versus $\omega_{x}$, when $\omega_{y}=0$, are shown in Figure 3. The scheme is in general less dissipative at $h=0.05$ as compared to $h=0.025$. Out of the eight combinations of values of $h$ and $k$, the scheme is 


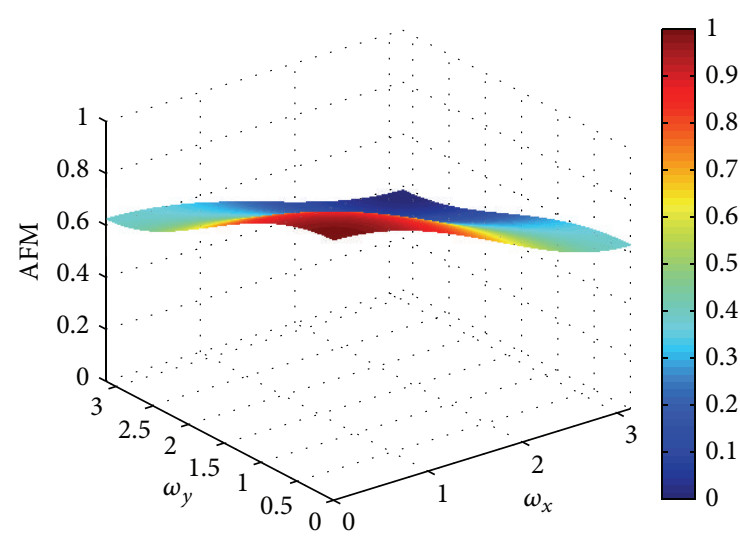

(a) $h=0.025, k=0.005$

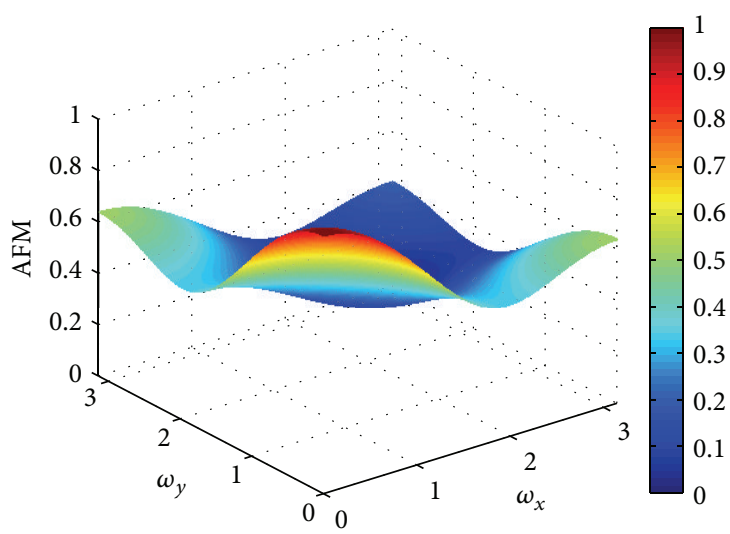

(c) $h=0.025, k=1 / 60$

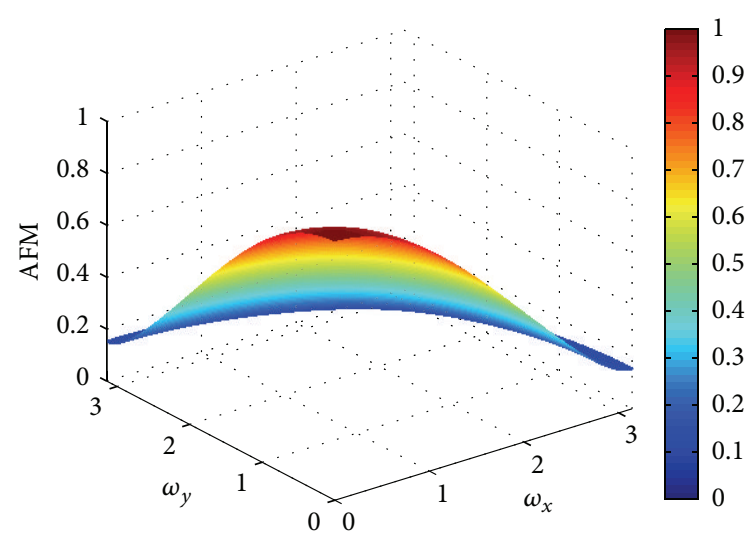

(b) $h=0.025, k=0.01$

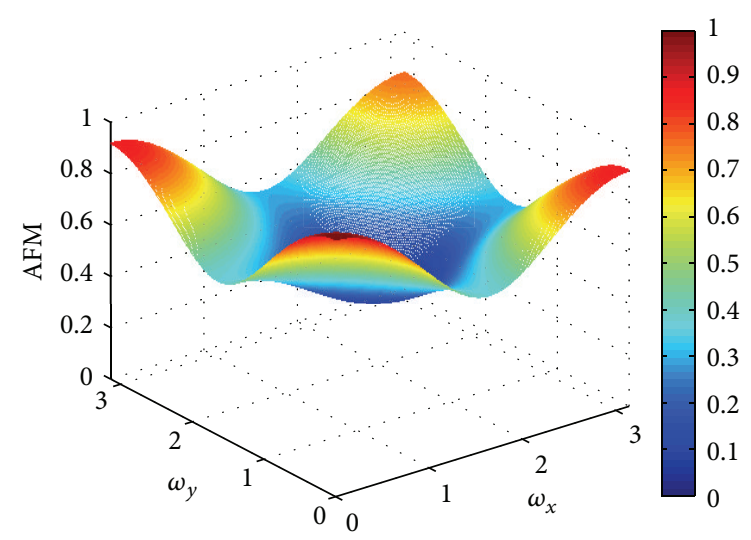

(d) $h=0.025, k=3 / 160$

FIGURE 1: Plots of modulus of amplification factor versus phase angle in $x$-direction, $\omega_{x}$, versus phase angle in $y$-direction, $\omega_{y}$, at $h=0.025$ with some different values of $k$ for the LOD Lax-Wendroff scheme.

least dissipative when $h=0.05$ and $k=0.005$ and it is most dissipative when $h=0.025$ and $k=0.01$.

Figures 4 and 5 show the 3D plots of the relative phase error versus $\omega_{x}$ versus $\omega_{y}$ for some different values of $h$ and $k$. Figure 6 shows the $2 \mathrm{D}$ plots of the relative phase error versus $\omega_{x}$, for the case $\omega_{y}=0$ at $h=0.025$ and $h=0.05$. For $h=0.025$, we observe phase lag behaviour at $k=0.01$ and $k=0.005$ and phase lead behaviour at $k=1 / 60$ and $k=3 / 160$. For $h=0.05$, we have phase lag behaviour at $k=0.005,0.01,0.02$, and 0.03 . The scheme is least dispersive when $h=0.05 ; k=0.03$ and $h=0.025 ; k=0.01$. In the following section, we consider the $\operatorname{LOD}(1,5)$ scheme.

\section{LOD $(1,5)$ Explicit Procedure}

In this procedure the following approximations are used in the first half time step [3]:

$$
\left.\frac{\partial u}{\partial t}\right|_{i, j} ^{n} \simeq \frac{u_{i, j}^{n+(1 / 2)}-u_{i, j}^{n}}{\Delta t}
$$

$$
\begin{aligned}
\left.\frac{\partial u}{\partial x}\right|_{i, j} ^{n} \simeq & \left.\frac{12 s_{x}+2 c_{x}^{2}-3 c_{x}-2}{12}\right)\left(\frac{u_{i+2, j}^{n}-u_{i, j}^{n}}{2 \Delta x}\right) \\
+ & \left(\frac{12 s_{x}+2 c_{x}^{2}+3 c_{x}-2}{12}\right)\left(\frac{u_{i, j}^{n}-u_{i-2, j}^{n}}{2 \Delta x}\right) \\
\left.\frac{\left.\partial^{2} u\right|^{n}}{\partial x^{2}}\right|_{i, j} & \left(\frac{-c_{x}^{4}+4 c_{x}^{2}-12 s_{x}^{2}-12 s_{x} c_{x}^{2}+8 s_{x}}{6 s_{x}}\right) \\
& \times\left(\frac{u_{i+1, j}^{n}-2 u_{i, j}^{n}+u_{i-1, j}^{n}}{12}\right) \\
& +\left(\frac{c_{x}^{n}-4 c_{x}^{2}+12 s_{x}^{2}+12 s_{x} c_{x}^{2}-2 s_{x}}{6 s_{x}}\right) \\
& \times\left(\frac{u_{i+2, j}^{n}-2 u_{i, j}^{n}+u_{i-2, j}^{n}}{4(\Delta x)^{2}}\right) .
\end{aligned}
$$




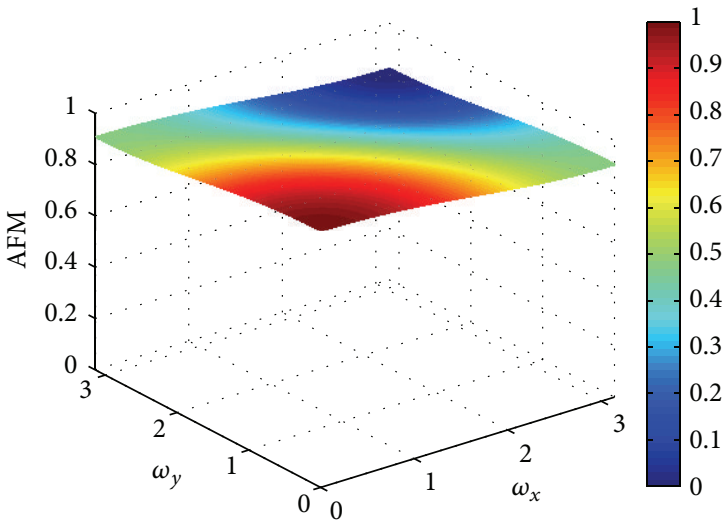

(a) $h=0.05, k=0.005$

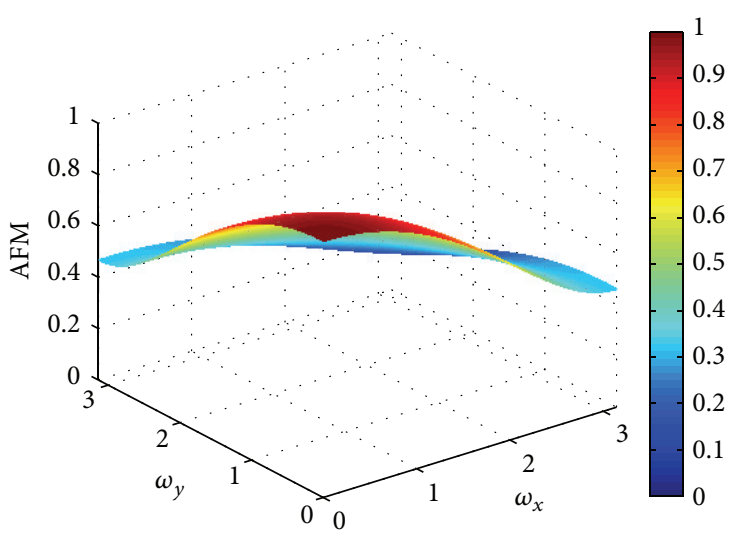

(c) $h=0.05, k=0.02$

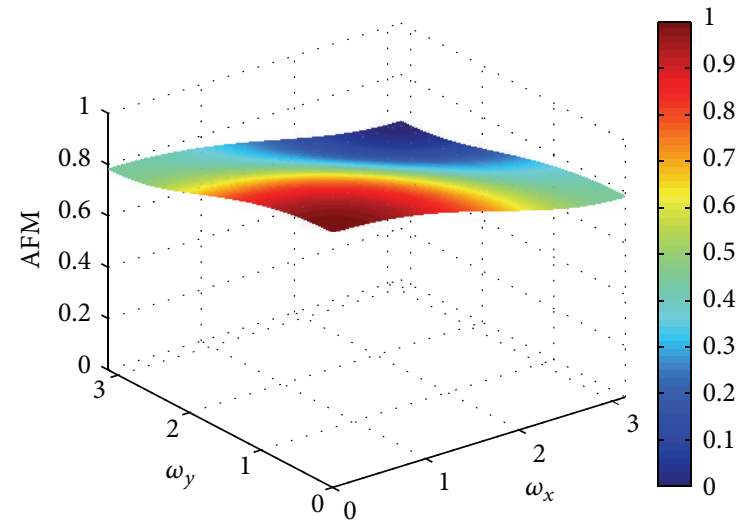

(b) $h=0.05, k=0.01$

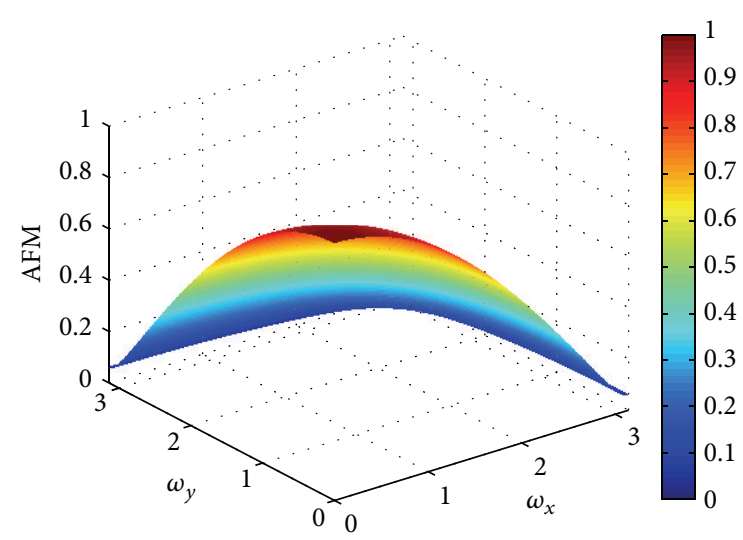

(d) $h=0.05, k=0.03$

Figure 2: Plots of modulus of amplification factor versus phase angle in $x$-direction, $\omega_{x}$, versus phase angle in $y$-direction, $\omega_{y}$, at $h=0.05$ with some different values of $k$ for the LOD Lax-Wendroff scheme.

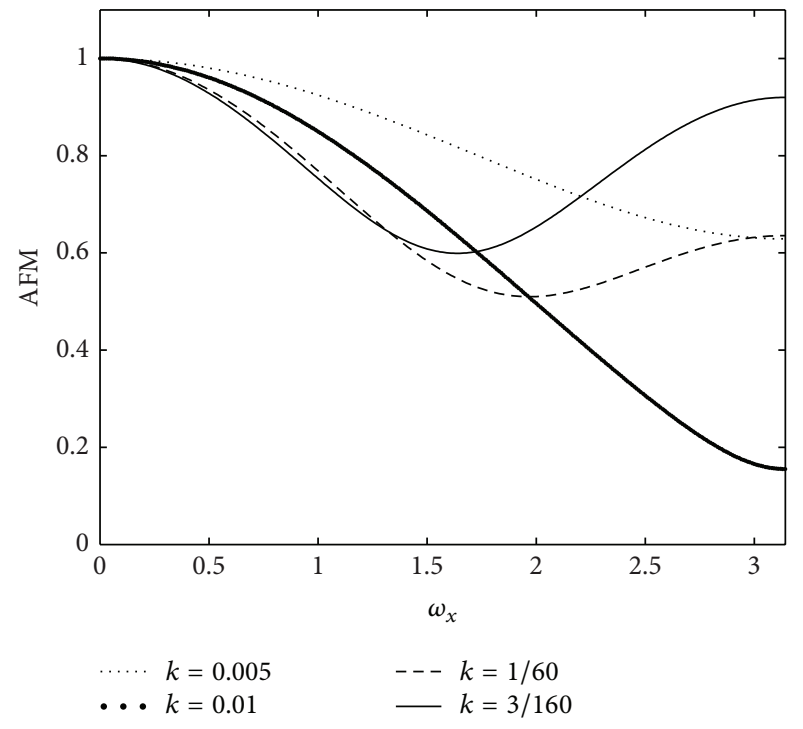

(a) $h=0.025$

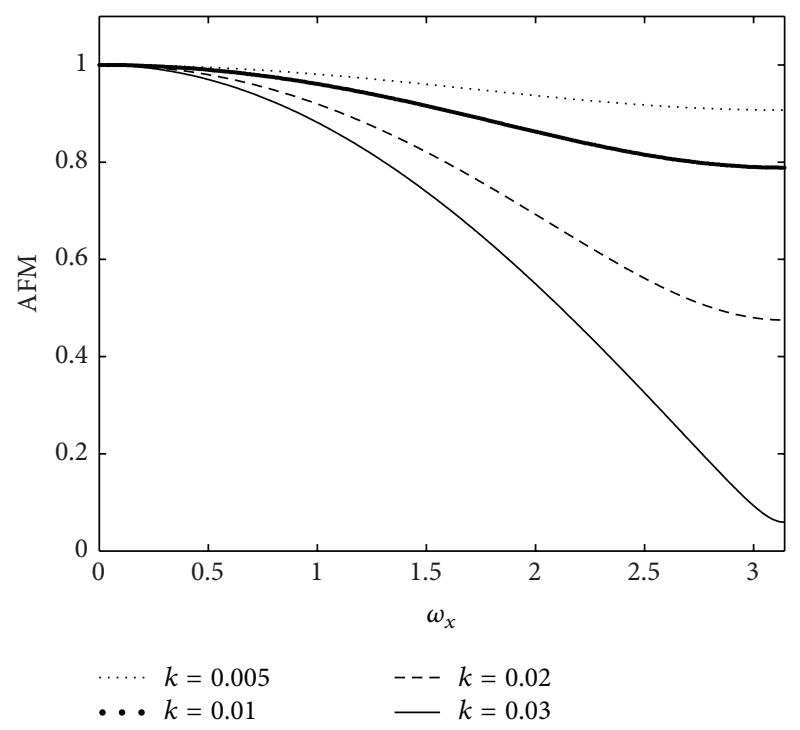

(b) $h=0.05$

FIGURE 3: Plots of modulus of amplification factor versus $\omega_{x}$ when $\omega_{y}=0$ at $h=0.025$ and $h=0.05$ with some different values of $k$ for the LOD Lax-Wendroff scheme. 


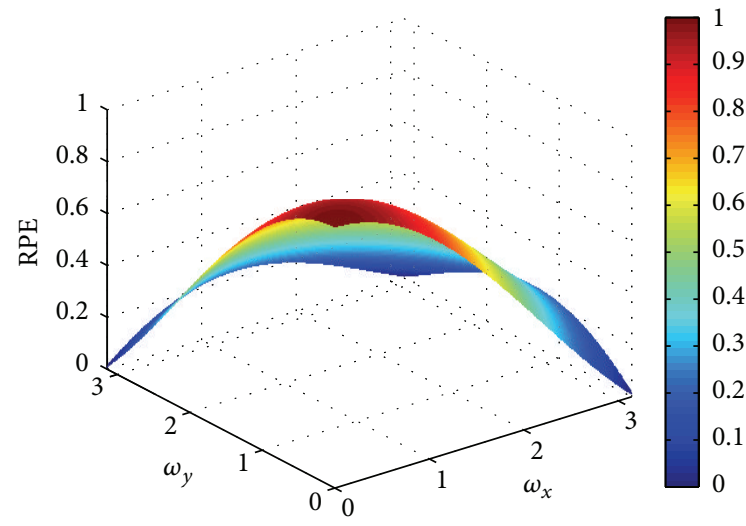

(a) $h=0.025, k=0.005$

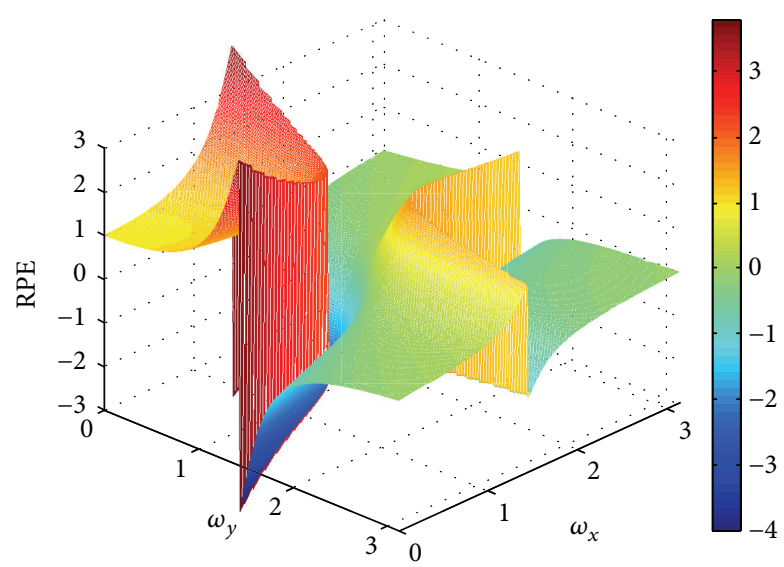

(c) $h=0.025, k=1 / 60$

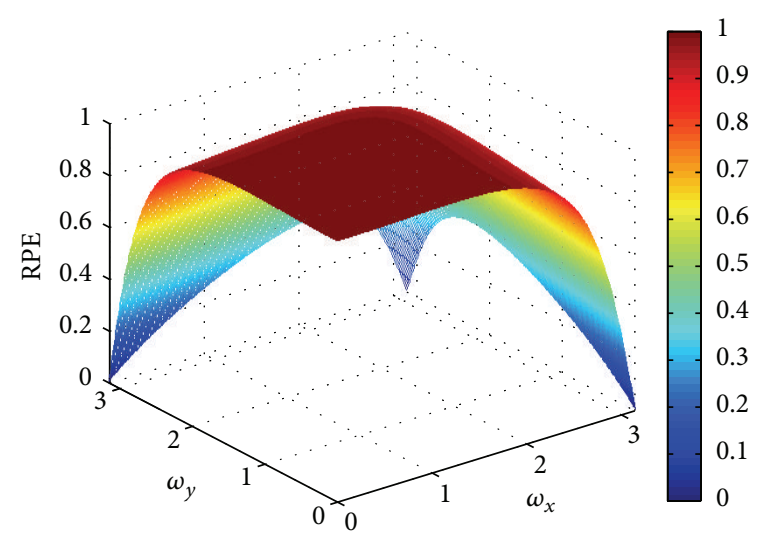

(b) $h=0.025, k=0.01$

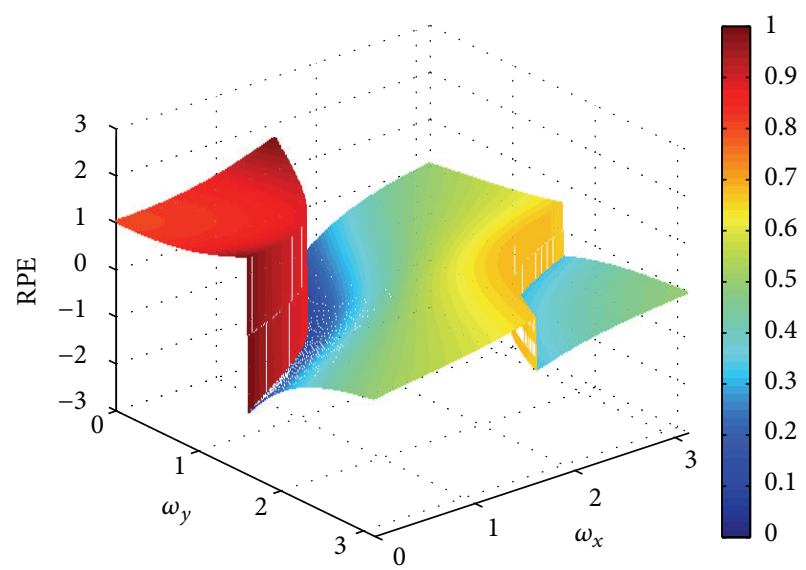

(d) $h=0.025, k=3 / 160$

FIGURE 4: Plots of relative phase error versus phase angle in $x$-direction $\left(\omega_{x}\right)$ versus phase angle in $y$-direction $(\omega-y)$, at $h=0.025$ with some different values of $k$ for the LOD Lax-Wendroff scheme.

On substituting (62) into (14), we get the following finite difference equation for the first half time step:

$$
\begin{aligned}
u_{i, j}^{n+1 / 2}= & A_{x} u_{i-2, j}^{n}+B_{x} u_{i-1, j}^{n}+C_{x} u_{i, j}^{n} \\
& +D_{x} u_{i+1, j}^{n}+E_{x} u_{i+2, j}^{n},
\end{aligned}
$$

where

$$
\begin{gathered}
A_{x}=\frac{1}{24}\left(12 s_{x}\left(s_{x}+c_{x}^{2}\right)+2 s_{x}\left(6 c_{x}-1\right)\right. \\
\left.+c_{x}\left(c_{x}-1\right)\left(c_{x}+1\right)\left(c_{x}+2\right)\right), \\
B_{x}=-\frac{1}{6}\left(12 s_{x}\left(s_{x}+c_{x}^{2}\right)+2 s_{x}\left(3 c_{x}-4\right)\right. \\
\left.\quad+c_{x}\left(c_{x}-2\right)\left(c_{x}+1\right)\left(c_{x}+2\right)\right), \\
C_{x}=\frac{1}{4}\left(12 s_{x}\left(s_{x}+c_{x}^{2}\right)-10 s_{x}\right. \\
\left.+\left(c_{x}-1\right)\left(c_{x}-2\right)\left(c_{x}+1\right)\left(c_{x}+2\right)\right),
\end{gathered}
$$

$$
\begin{aligned}
D_{x}=-\frac{1}{6}( & 12 s_{x}\left(s_{x}+c_{x}^{2}\right)-2 s_{x}\left(3 c_{x}+4\right) \\
& \left.+c_{x}\left(c_{x}-2\right)\left(c_{x}-1\right)\left(c_{x}+2\right)\right), \\
E_{x}=\frac{1}{24}( & 12 s_{x}\left(s_{x}+c_{x}^{2}\right)-2 s_{x}\left(6 c_{x}+1\right) \\
& \left.+c_{x}\left(c_{x}-1\right)\left(c_{x}+1\right)\left(c_{x}-2\right)\right) .
\end{aligned}
$$

The following approximations are used in the second half time step:

$$
\begin{gathered}
\left.\frac{\partial u}{\partial t}\right|_{i, j} ^{n+(1 / 2)} \simeq \frac{u_{i, j}^{n+1}-u_{i, j}^{n+(1 / 2)}}{\Delta t}, \\
\left.\frac{\partial u}{\partial x}\right|_{i, j} ^{n+(1 / 2)} \\
\simeq\left(\frac{12 s_{y}+2 c_{y}^{2}-3 c_{y}-2}{12}\right)\left(\frac{u_{i, j+2}^{n+(1 / 2)}-u_{i, j}^{n+(1 / 2)}}{2 \Delta y}\right)
\end{gathered}
$$




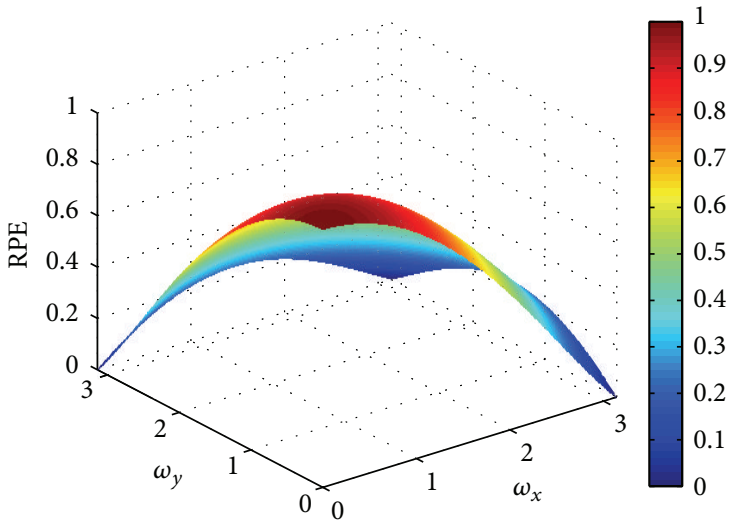

(a) $h=0.05, k=0.005$

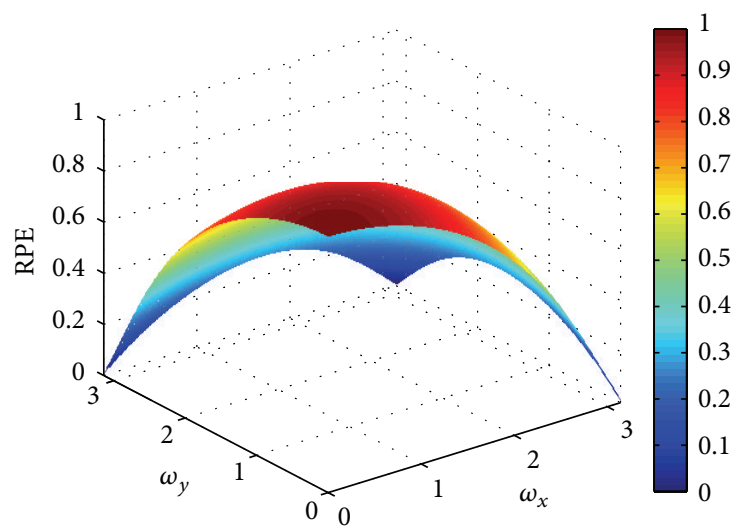

(c) $h=0.05, k=0.02$

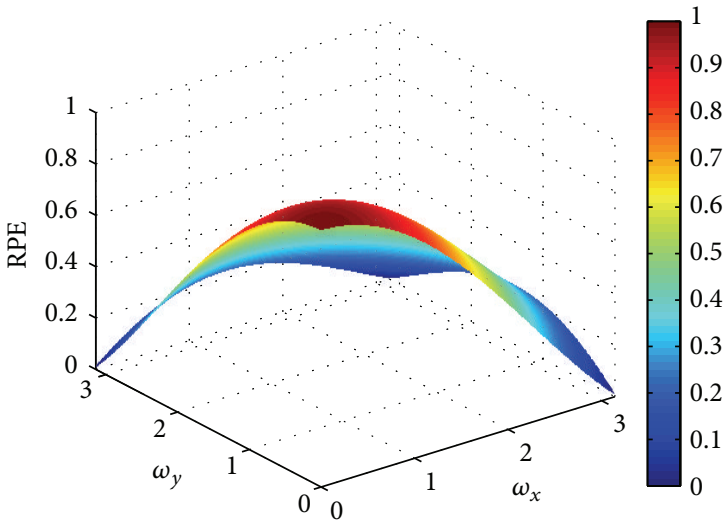

(b) $h=0.05, k=0.01$

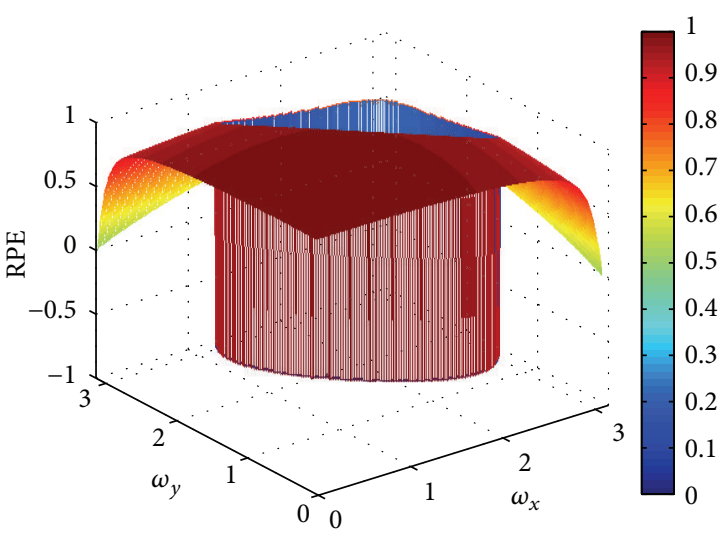

(d) $h=0.05, k=0.03$

Figure 5: Plots of relative phase error versus $\omega_{x}$ versus $\omega_{y}$, at $h=0.05$ with some different values of $k$ for the LOD Lax-Wendroff scheme.

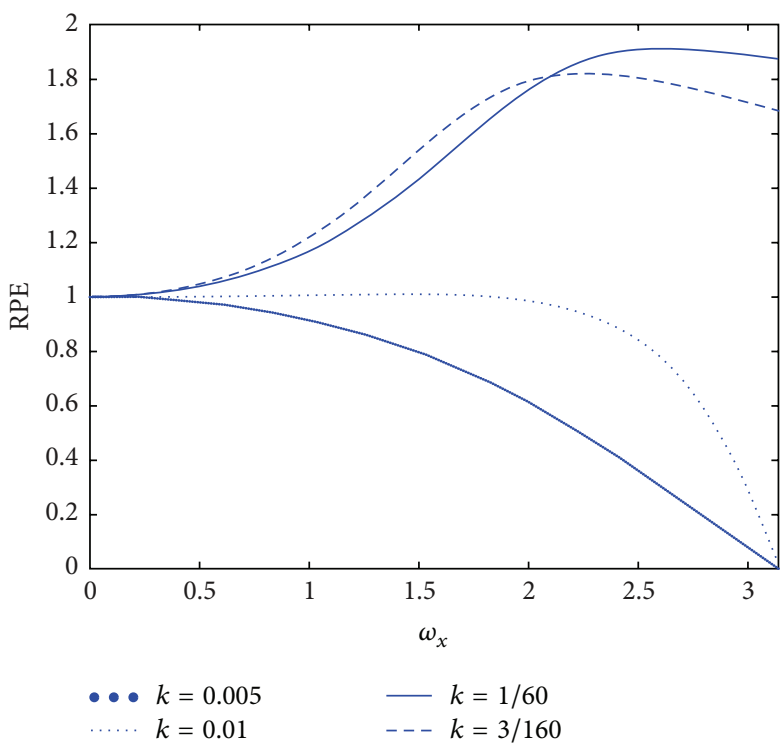

(a) $h=0.025$

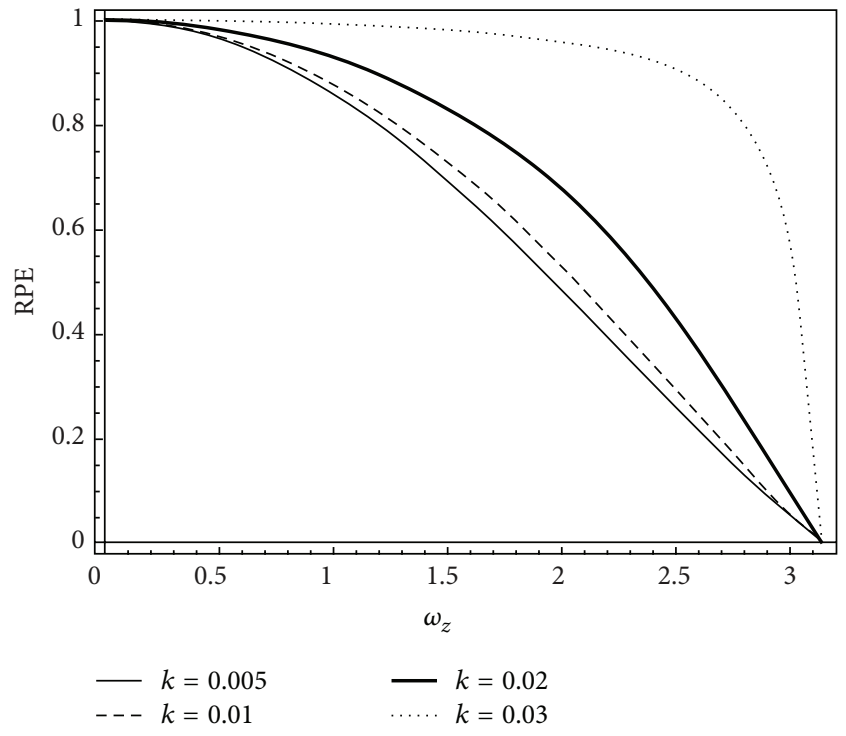

(b) $h=0.05$

FIgURE 6: Plots of relative phase error versus $\omega_{x}$ when $\omega_{y}=0$ at $h=0.025$ and $h=0.05$ with some different values of $k$ for the LOD Lax-Wendroff scheme. 


$$
\begin{aligned}
+\left(\frac{12 s_{y}+2 c_{y}^{2}+3 c_{y}-2}{12}\right)\left(\frac{u_{i, j}^{n+(1 / 2)}-u_{i, j-2}^{n+(1 / 2)}}{2 \Delta y}\right) \\
-\left(\frac{c_{y}^{2}+6 s_{y}-4}{12}\right)\left(\frac{u_{i, j+1}^{n+(1 / 2)}-u_{i, j-1}^{n+(1 / 2)}}{2 \Delta y}\right), \\
\left.\frac{\partial^{2} u}{\partial y^{2}}\right|_{i, j} ^{n+(1 / 2)} \simeq\left(\frac{-c_{y}^{4}+4 c_{y}^{2}-12 s_{y}^{2}-12 s_{y} c_{y}^{2}+8 s_{y}}{6 s_{y}}\right) \\
\times\left(\frac{u_{i, j+1}^{n+(1 / 2)}-2 u_{i, j}^{n+(1 / 2)}+u_{i, j-1}^{n+(1 / 2)}}{(\Delta y)^{2}}\right) \\
+\left(\frac{c_{y}^{4}-4 c_{y}^{2}+12 s_{y}^{2}+12 s_{y} c_{y}^{2}-2 s_{y}}{6 s_{y}}\right) \\
\times\left(\frac{u_{i, j+2}^{n+(1 / 2)}-2 u_{i, j}^{n+(1 / 2)}+u_{i, j-2}^{n+(1 / 2)}}{4(\Delta y)^{2}}\right) .
\end{aligned}
$$

Then on substituting (65) into (15), we get the following difference equation for the second half time step:

$$
\begin{aligned}
u_{i, j}^{n+1}= & A_{y} u_{i, j-2}^{n+(1 / 2)}+B_{y} u_{i, j-1}^{n+(1 / 2)}+C_{y} u_{i, j}^{n+(1 / 2)} \\
& +D_{y} u_{i, j+1}^{n+(1 / 2)}+E_{y} u_{i, j+2}^{n+(1 / 2)}
\end{aligned}
$$

where

$$
\begin{gathered}
A_{y}=\frac{1}{24}\left(12 s_{y}\left(s_{y}+c_{y}^{2}\right)+2 s_{y}\left(6 c_{y}-1\right)\right. \\
\left.+c_{y}\left(c_{y}-1\right)\left(c_{y}+1\right)\left(c_{y}+2\right)\right), \\
B_{y}=-\frac{1}{6}\left(12 s_{y}\left(s_{y}+c_{y}^{2}\right)+2 s_{y}\left(3 c_{y}-4\right)\right. \\
\left.+c_{y}\left(c_{y}-2\right)\left(c_{y}+1\right)\left(c_{y}+2\right)\right), \\
C_{y}=\frac{1}{4}\left(12 s_{y}\left(s_{y}+c_{y}^{2}\right)-10 s_{y}\right. \\
\left.+\left(c_{y}-1\right)\left(c_{y}-2\right)\left(c_{y}+1\right)\left(c_{y}+2\right)\right), \\
D_{y}=-\frac{1}{6}\left(12 s_{y}\left(s_{y}+c_{y}^{2}\right)-2 s_{y}\left(3 c_{y}+4\right)\right. \\
\left.+c_{y}\left(c_{y}-2\right)\left(c_{y}-1\right)\left(c_{y}+2\right)\right), \\
E_{y}=\frac{1}{24}\left(12 s_{y}\left(s_{y}+c_{y}^{2}\right)-2 s_{y}\left(6 c_{y}+1\right)\right. \\
\left.+c_{y}\left(c_{y}-1\right)\left(c_{y}+1\right)\left(c_{y}-2\right)\right) .
\end{gathered}
$$

The complete $\operatorname{LOD}(1,5)$ scheme is given by

$$
\begin{aligned}
u_{i, j}^{n+1}= & A_{y} A_{x} u_{i-2, j-2}^{n}+A_{y} B_{x} u_{i-1, j-2}^{n}+A_{y} C_{x} u_{i, j-2}^{n} \\
& +A_{y} D_{x} u_{i+1, j-2}^{n}+A y E x u_{i+2, j-2}^{n} \\
& +B_{y} A_{x} u_{i-2, j-1}^{n}+B_{y} B_{x} u_{i-1, j-1}^{n}+B_{y} C_{x} u_{i, j-1}^{n} \\
& +B_{y} D_{x} u_{i+1, j-1}^{n}+B_{y} E_{x} u_{i+2, j-1}^{n} \\
& +C_{y} A_{x} u_{i-2, j}^{n}+C_{y} B_{x} u_{i-1, j}^{n}+C_{y} C_{x} u_{i, j}^{n} \\
& +C_{y} D_{x} u_{i+1, j}^{n}+C_{y} E_{x} u_{i+2, j}^{n} \\
& +D_{y} A_{x} u_{i-2, j+1}^{n}+D_{y} B_{x} u_{i-1, j+1}^{n}+D_{y} C_{x} u_{i, j+1}^{n} \\
& +D_{y} D_{x} u_{i+1, j+1}^{n}+D_{y} E_{x} u_{i+2, j+1}^{n} \\
& +E_{y} A_{x} u_{i-2, j+2}^{n}+E_{y} B_{x} u_{i-1, j+2}^{n}+E_{y} C_{x} u_{i, j+2}^{n} \\
& +E_{y} D_{x} u_{i+1, j+2}^{n}+E_{y} E_{x} u_{i+2, j+2}^{n} .
\end{aligned}
$$

The Taylor series expansion of the terms on the right hand side of (68) about $u_{i, j}^{n}$ is given as follows:

$$
\begin{aligned}
u_{i+a, j+b}^{n}= & u+a h u_{x}+b h u_{y}+\frac{h^{2}}{2}\left(a^{2} u_{x x}+2 a b u_{x y}+b^{2} u_{y y}\right) \\
+ & \frac{h^{3}}{6}\left(a^{3} u_{x x x}+3 a^{2} b u_{x x y}+3 a b^{2} u_{x y y}+b^{3} u_{y y y}\right) \\
+ & \frac{h^{4}}{24}\left(a^{4} u_{x x x x}+4 a^{3} b u_{x x x y}+6 a^{2} b^{2} u_{x x y y}\right. \\
& \left.+4 a b^{3} u_{x y y y}+b^{4} u_{y y y y}\right) \\
+ & \frac{h^{5}}{120}\left(a^{5} u_{x x x x x}+5 a^{4} b u_{x x x x y}\right. \\
& +10 a^{3} b^{2} u_{x x x y y}+10 a^{2} b^{3} u_{x x y y y} \\
& \left.+5 a b^{4} u_{x y y y y}+b^{5} u_{y y y y y}\right)+\cdots
\end{aligned}
$$

for $a=-2,-1,0,1,2$ and $b=-2,-1,0,1,2$.

We obtain the following modified equation:

$$
\begin{aligned}
& u_{t}+\beta_{x} u_{x}+\beta_{y} u_{y}= \alpha_{x} u_{x x}+\alpha_{y} u_{y y} \\
&+\frac{\beta_{x} h^{4}}{120}\left(60 s_{x}^{2}+20 s_{x} c_{x}^{2}+c_{x}^{4}\right. \\
&\left.-5 c_{x}^{2}+4-30 s_{x}\right) u_{x x x x x} \\
&+\frac{\beta_{y} h^{4}}{120}\left(60 s_{y}^{2}+20 s_{y} c_{y}^{2}+c_{y}^{4}\right. \\
&\left.-5 c_{y}^{2}+4-30 s_{y}\right) u_{y y y y y}+\cdots
\end{aligned}
$$


The scheme is essentially dispersive as the leading error terms are dispersive in nature due to the presence of the oddorder derivative terms $u_{x x x x x}$ and $u_{y y y y y}$. Also, the scheme is consistent and it is fourth order accurate in space. by

The amplification factor of the $\operatorname{LOD}(1,5)$ scheme is given

$$
\begin{aligned}
\xi=A_{y}[ & A_{x} \exp \left(I\left(-2 \omega_{x}-2 \omega_{y}\right)\right)+B_{x} \exp \left(I\left(-\omega_{x}-2 \omega_{y}\right)\right) \\
& +C_{x} \exp \left(-2 I \omega_{y}\right) \\
& \left.+D_{x} \exp \left(I\left(\omega_{x}-2 \omega_{y}\right)\right)+E_{x} \exp \left(I\left(2 \omega_{x}-2 \omega_{y}\right)\right)\right] \\
+B_{y}[ & A_{x} \exp \left(I\left(-2 \omega_{x}-\omega_{y}\right)\right)+B_{x} \exp \left(I\left(-\omega_{x}-\omega_{y}\right)\right) \\
& +C_{x} \exp \left(-I \omega_{y}\right)+D_{x} \exp \left(I\left(\omega_{x}-\omega_{y}\right)\right) \\
& \left.+E_{x} \exp \left(I\left(2 \omega_{x}-\omega_{y}\right)\right)\right] \\
+C_{y}[ & A_{x} \exp \left(-2 I \omega_{x}\right)+B_{x} \exp \left(-I \omega_{x}\right) \\
& \left.+C_{x}+D_{x} \exp \left(I \omega_{x}\right)+E_{x} \exp \left(2 I \omega_{x}\right)\right] \\
+D_{y} & {\left[A_{x} \exp \left(I\left(-2 \omega_{x}+\omega_{y}\right)\right)+B_{x} \exp \left(-I\left(\omega_{x}+\omega_{y}\right)\right)\right.} \\
& +C_{x} \exp \left(I \omega_{y}\right)+D_{x} \exp \left(I\left(\omega_{x}+\omega_{y}\right)\right) \\
& \left.+E_{x} \exp \left(I\left(2 \omega_{x}+\omega_{y}\right)\right)\right] \\
& \left.+E_{x} \exp \left(I\left(2 \omega_{x}+2 \omega_{y}\right)\right)\right] . \\
+E_{y}[ & A_{x} \exp \left(I\left(-2 \omega_{x}+2 \omega_{y}\right)\right) \\
& +B_{x} \exp \left(I\left(-\omega_{x}+2 \omega_{y}\right)\right)+C_{x} \exp \left(2 I \omega_{y}\right) \\
& +D_{x} \exp \left(I\left(\omega_{x}+2 \omega_{y}\right)\right) \\
& \\
& \exp (1)
\end{aligned}
$$

We consider $c_{x}=c_{y}=c$ and $s_{x}=s_{y}=s$. We use the Von Neumann stability analysis and the approach of Hindmarsh et al. [16] to obtain the stability region. When $\omega_{x}=\omega_{y}=\pi$, on simplification of (71), we get

$$
|\xi|=\frac{1}{9}\left(3-8 c^{2}+2 c^{4}-16 s+24 s c^{2}+24 s^{2}\right)^{2} .
$$

For stability, we must have

$$
\frac{1}{9}\left(3-8 c^{2}+2 c^{4}-16 s+24 s c^{2}+24 s^{2}\right)^{2} \leq 1 .
$$

We consider (71) and for $\omega_{x} \rightarrow 0$ and $\omega_{y} \rightarrow 0$ we use the following approximations:

$$
\begin{aligned}
& \cos \left(\omega_{x}\right) \simeq 1-\frac{\omega_{x}^{2}}{2}, \quad \cos \left(\omega_{y}\right) \simeq 1-\frac{\omega_{y}^{2}}{2}, \\
& \cos \left(2 \omega_{x}\right) \simeq 1-2 \omega_{x}^{2}, \quad \cos \left(2 \omega_{y}\right) \simeq 1-2 \omega_{y}^{2}, \\
& \sin \left(\omega_{x}\right) \simeq \omega_{x}, \quad \sin \left(\omega_{y}\right) \simeq \omega_{y}, \\
& \sin \left(2 \omega_{x}\right) \simeq 2 \omega_{x}, \quad \sin \left(2 \omega_{y}\right) \simeq 2 \omega_{y} .
\end{aligned}
$$

We thus have

$$
\begin{aligned}
|\xi|^{2} \simeq & 1-2 s\left(\omega_{x}^{2}+\omega_{y}^{2}\right)+4 s^{2} \omega_{x}^{2} \omega_{y}^{2} \\
& +\left(-2 s^{3} \omega_{y}^{2}+s c^{2}+\frac{1}{4} c^{4}-2 c^{2} s^{2} \omega_{y}^{2}-\frac{1}{2} s c^{4} \omega_{y}^{2}+s^{2}\right) \omega_{x}^{4} \\
& +\left(s^{4}+\frac{1}{16} c^{8}+\frac{3}{2} s^{2} c^{4}+\frac{1}{2} s c^{6}+2 s^{3} c^{2}\right) \omega_{x}^{4} \omega_{y}^{4} \\
& +\left(-\frac{1}{2} c^{4} s \omega_{x}^{2}-2 c^{2} s^{2} \omega_{x}^{2}+\frac{1}{4} c^{4}+s^{2}-2 s^{3} \omega_{x}^{2}+c^{2} s\right) \omega_{y}^{4} .
\end{aligned}
$$

On neglecting higher order terms, we have

$$
|\xi|^{2} \simeq 1-2 s\left(\omega_{x}^{2}+\omega_{y}^{2}\right) .
$$

Thus, the numerical method is stable if $-2 s \leq 0$. Therefore, we have

$$
s \geq 0
$$

When $h=0.025$, we have $c=32 k$ and $s=16 k$. Using (73) and (77), we get

$$
\begin{gathered}
\frac{1}{9}\left(2097152 k^{4}+393216 k^{3}-2048 k^{2}-256 k+3\right)^{2} \leq 1 \\
k \geq 0,
\end{gathered}
$$

respectively. On combining (78) and (79), we get the stability region when $h=0.025$ for the $\operatorname{LOD}(1,5)$ procedure as

$$
0 \leq k \leq 0.026288 \text {. }
$$

When $h=0.05$, we have $c=16 k$ and $s=4 k$ and the modulus of the amplification factor is given by

$$
|\xi|=\frac{1}{9}\left(131072 k^{4}+24576 k^{3}-1664 k^{2}-64 k+3\right)^{2} .
$$

From (77) and (81), we obtain the stability region for the LOD $(1,5)$ procedure when $h=0.05$ as

$$
0 \leq k \leq 0.073865 \text {. }
$$

We analyse the spectral analysis; for $h=0.025$, we choose $k=0.005,0.01,0.02,0.025$ and for $h=0.05$, we choose $k=$ $0.01,0.02,0.03$ and 0.05 .

$3 \mathrm{D}$ plots of the modulus of the amplification factor versus phase angle in $x$-direction versus phase angle in $y$-direction at two values of $h$, namely, 0.025 and 0.05 , at some different values of $k$ are shown in Figures 7 and 8. 2D plots of the modulus of the amplification factor versus $\omega_{x}$ when $\omega_{y}=$ 0 are illustrated in Figure 9. The scheme is in general less dissipative at $h=0.05$ as compared to $h=0.025$. Out of the eight combinations of $h$ and $k$ values, the scheme is least dissipative when $h=0.05$ and $k=0.01$.

Figures 10 and 11 show the $3 \mathrm{D}$ plots of the relative phase error versus $\omega_{x}$ versus $\omega_{y}$ for some different values of $h$ and $k$. Figure 12 shows the $2 \mathrm{D}$ plots of the relative phase error versus $\omega_{x}$, for the case $\omega_{y}=0$ at $h=0.025$ and $h=0.05$, respectively. 


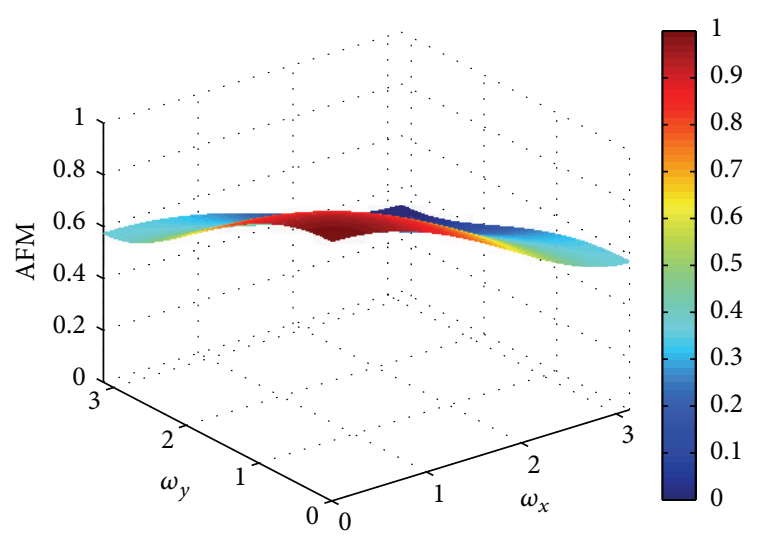

(a) $h=0.025, k=0.005$

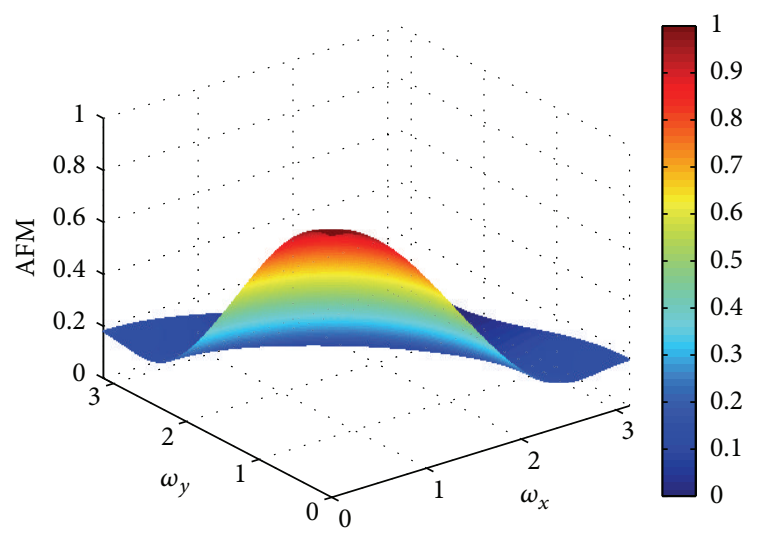

(c) $h=0.025, k=0.02$

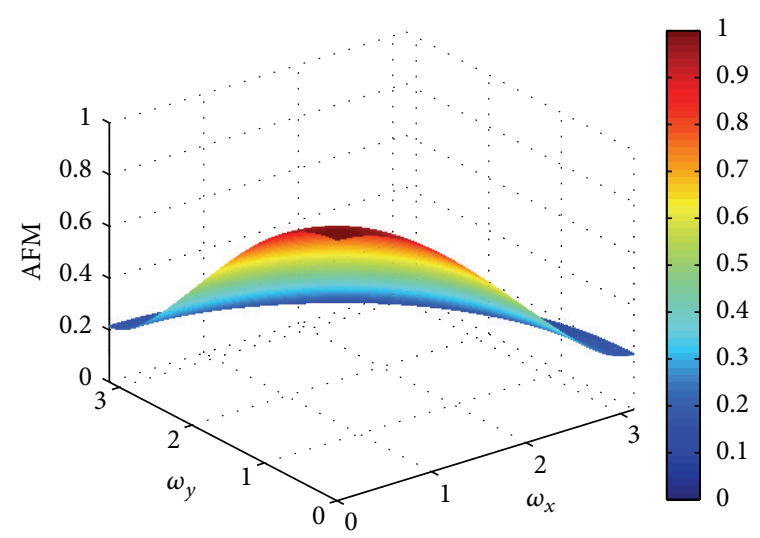

(b) $h=0.025, k=0.01$

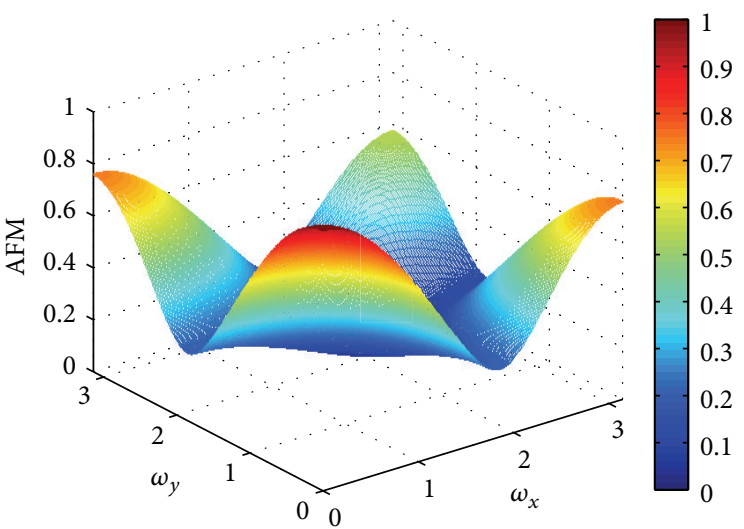

(d) $h=0.025, k=0.025$

FIGURE 7: Plots of modulus of amplification factor versus phase angle in $x$-direction $\left(\omega_{x}\right)$ versus phase angle in $y$-direction $\left(\omega_{y}\right)$, at $h=0.025$ for some different values of $k$ for the $\operatorname{LOD}(1,5)$ procedure.

We observe that the scheme is least dispersive when $h=0.05$ and $k=0.05$ and most dispersive when $h=0.025$ and $k=0.025$. We have phase lead when $h=0.05$ and $k=0.05$ and there is phase lag for the other seven combinations of $h$ and $k$. In the following section, we present the results of the numerical experiment described in Section 3.2.

\section{Numerical Results}

6.1. LOD Lax-Wendroff Procedure. The results of the numerical experiment at some values of $k$ using the LOD LaxWendroff procedure at $T=0.3$ at $h=0.025$ and $h=0.05$ are shown in Tables 1 and 2, respectively. We observe that the dispersion error is significantly greater than the dissipation error. Out of the five combinations of values of $k$ and $h$, we observe that the dispersion error and total mean square error are both least when $h=0.05$ and $k=0.03$ and are both greatest when $h=0.05$ and $k=0.0025$.

6.2. $\operatorname{LOD}(1,5)$ Procedure. The results of the numerical experiment using the $\operatorname{LOD}(1,5)$ procedure at $T=0.3$ for $h=$ 0.025 and $h=0.05$ are shown in Tables 3 and 4, respectively. Out of the 12 combinations of values of $k$ and $h$, we observe that the dissipation, dispersion error, and error rate are all least when $h=0.05$ and $k=0.05$. Also, the dissipation and dispersion errors and error rate are greatest when $h=0.05$ and $k=0.0625$. We observe that at $h=0.025$ the total mean square error, dispersion error, and dissipation error are not much affected by the values of $k$. Also, the dispersion error is greater than the at all values of $h$ and $k$ considered.

\section{Optimization}

In this section, we obtain the optimal value of $k$ at $h=0.025$ that minimizes the dispersion error for the two time-splitting procedures.

Since the partial differential equation we consider is slightly dissipative and also we observe from the numerical experiments carried out that the dissipative errors are much less than the dispersive errors, we choose to minimize the square of the dispersion error of the two splitting schemes.

7.1. Proposed Techniques of Optimization. Tam and Webb [18], Bogey and Bailly [19], and Hixon [20] among others have implemented techniques which enable coefficients to be determined in numerical schemes specifically designed for computational aeroacoustics. We now describe briefly how Tam and Webb [18] and Bogey and Bailly [19] define their 


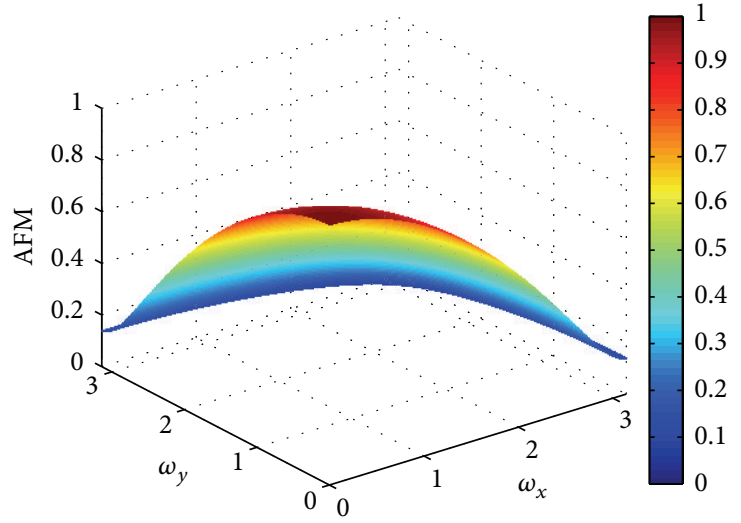

(a) $h=0.05, k=0.01$

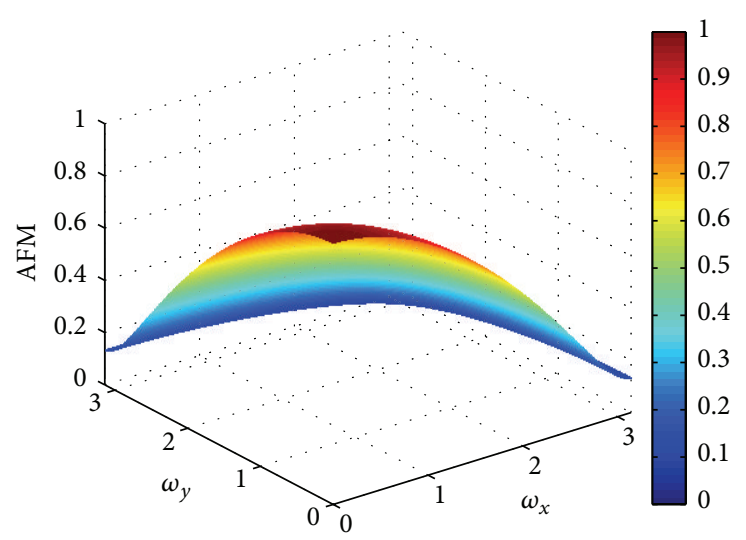

(c) $h=0.05, k=0.03$

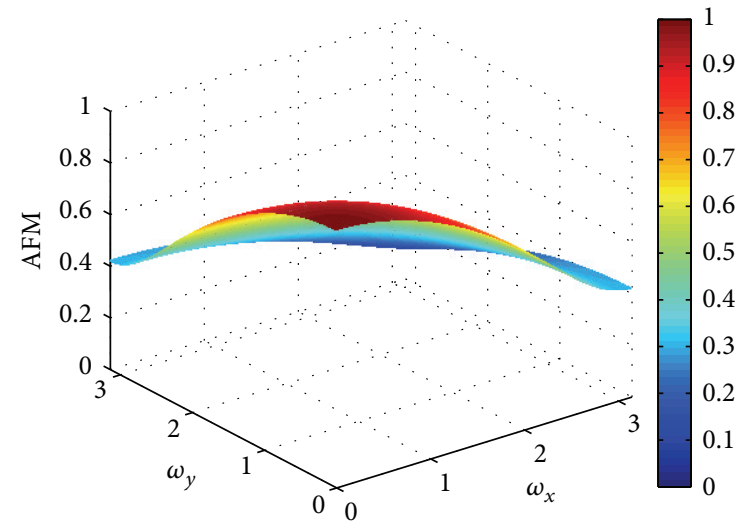

(b) $h=0.05, k=0.02$

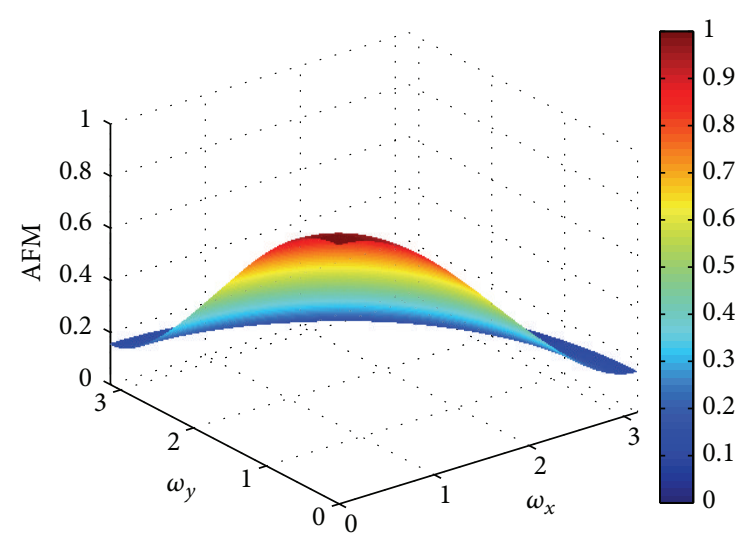

(d) $h=0.05, k=0.05$

FIGURE 8: Plots of modulus of amplification factor versus phase angle in $x$-direction $\left(\omega_{x}\right)$ versus phase angle in $y$-direction $\left(\omega_{y}\right)$ at $h=0.05$ for some different values of $k$ for the $\operatorname{LOD}(1,5)$ procedure.

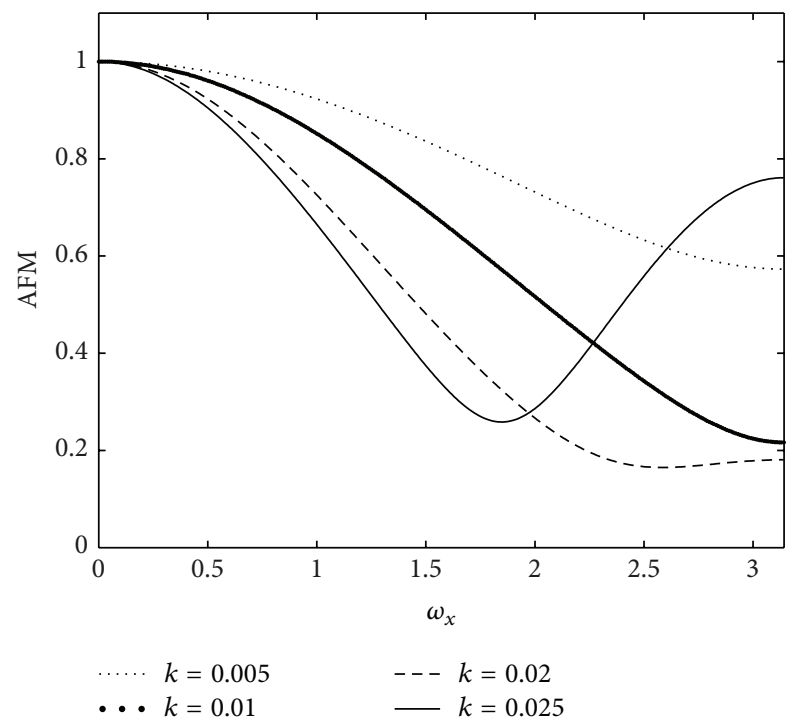

(a) $h=0.025$

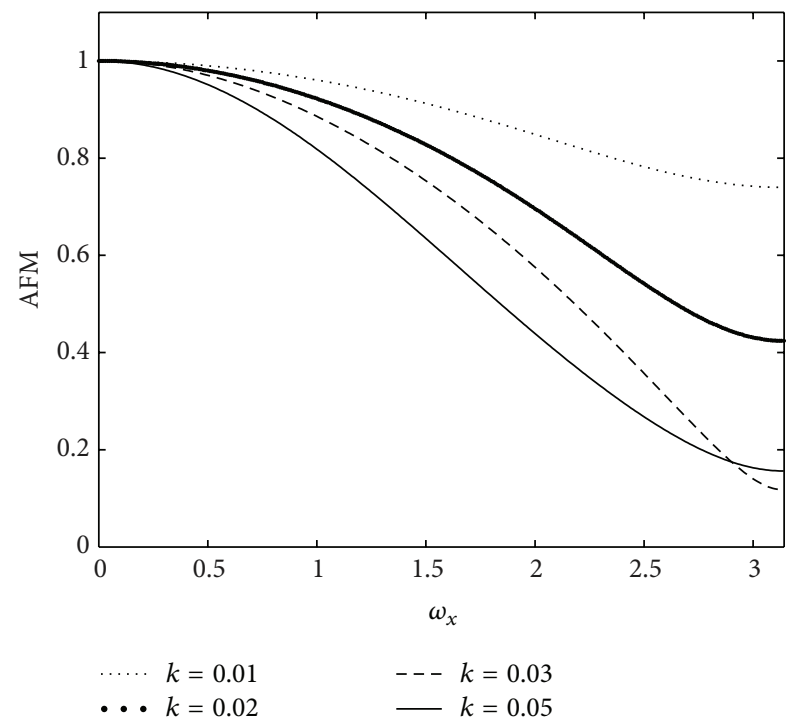

(b) $h=0.05$

Figure 9: Plots of modulus of amplification factor versus $\omega_{x}$ when $\omega_{y}=0$ at $h=0.025$ and $h=0.05$ with some different values of $k$ for the LOD $(1,5)$ procedure. 


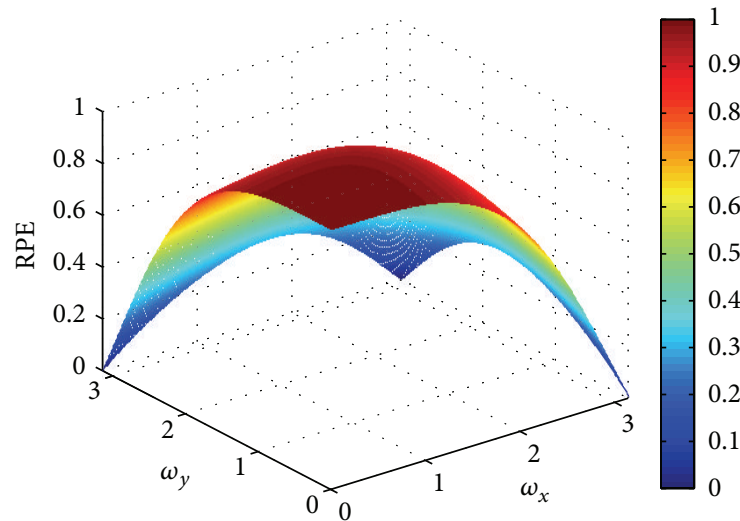

(a) $h=0.025, k=0.005$

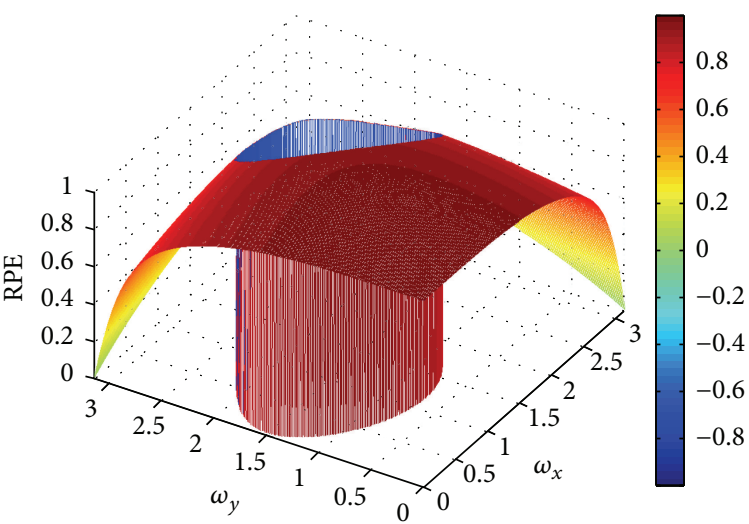

(c) $h=0.025, k=0.02$

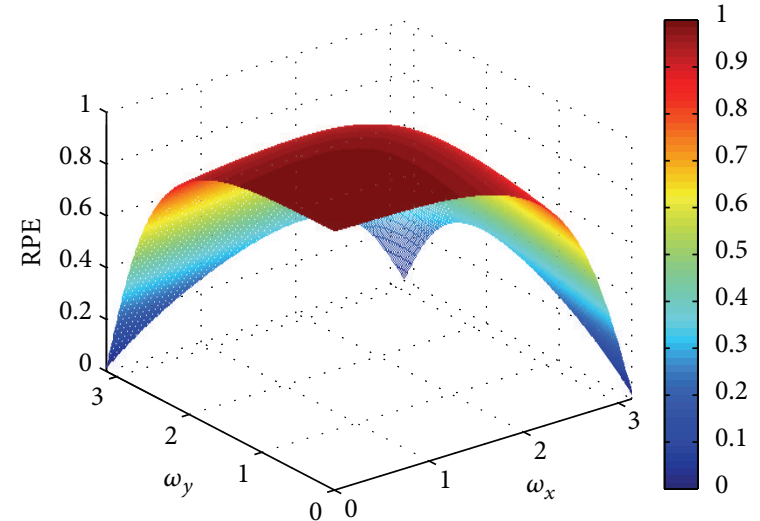

(b) $h=0.025, k=0.01$

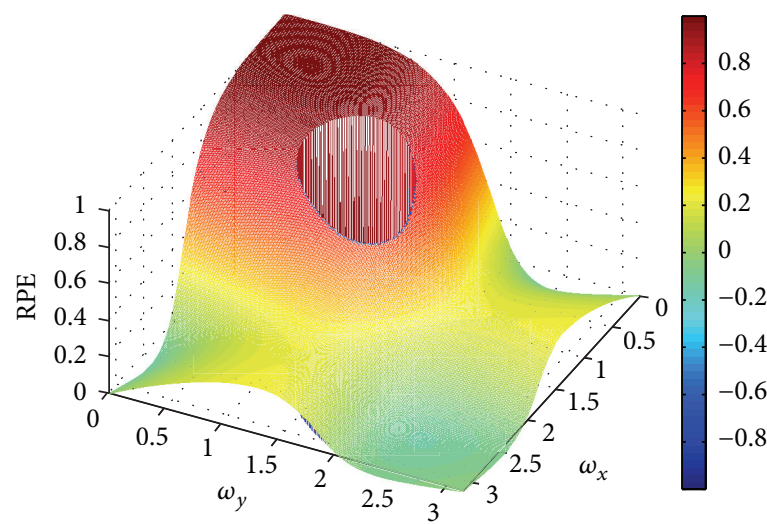

(d) $h=0.025, k=0.025$

Figure 10: 3D plots of relative phase error versus $\omega_{x}$ versus $\omega_{y}$ at $h=0.025$ at some different values of $k$ for the LOD $(1,5)$ procedure.

TABLE 1: Errors obtained from LOD Lax-Wendroff at $h=0.025$.

\begin{tabular}{lccccc}
\hline$k$ & CFL & $E_{\text {num }}$ & Total mean square error & DISP. ERROR & DISS. ERROR \\
\hline 0.0025 & 0.08 & $1.2043 \times 10^{-3}$ & $1.0930 \times 10^{-5}$ & $1.0602 \times 10^{-5}$ & $2.5787 \times 10^{-7}$ \\
0.005 & 0.16 & $9.4236 \times 10^{-4}$ & $7.4519 \times 10^{-6}$ & $4.2355 \times 10^{-6}$ & $2.4370 \times 10^{-7}$ \\
0.01 & 0.32 & $4.1557 \times 10^{-4}$ & $4.1970 \times 10^{-6}$ & $2.022 \times 10^{-6}$ & $1.6465 \times 10^{-7}$ \\
$3 / 160$ & 0.6 & $1.7126 \times 10^{-3}$ & $2.0913 \times 10^{-5}$ & $10^{-5}$ & $5.5626 \times 10^{-8}$ \\
$1 / 60$ & $8 / 15$ & $1.3626 \times 10^{-3}$ & $1.3761 \times 10^{-5}$ & $1.3675 \times 10^{-5}$ & $8.3438 \times 10^{-8}$ \\
\hline
\end{tabular}

TABLE 2: Errors obtained from LOD Lax-Wendroff at $h=0.05$.

\begin{tabular}{lccccc}
\hline$k$ & CFL & $E_{\text {num }}$ & Total mean square error & DISP. ERROR & DISS. ERROR \\
\hline 0.0025 & 0.04 & $1.3398 \times 10^{-3}$ & $4.1073 \times 10^{-5}$ & $4.1056 \times 10^{-5}$ & $2.1203 \times 10^{-8}$ \\
0.005 & 0.08 & $1.2483 \times 10^{-3}$ & $3.5560 \times 10^{-5}$ & $3.5562 \times 10^{-5}$ & $3.5412 \times 10^{-9}$ \\
0.01 & 0.16 & $1.0547 \times 10^{-3}$ & $2.5261 \times 10^{-5}$ & $2.5255 \times 10^{-5}$ & $8.1665 \times 10^{-9}$ \\
$1 / 60$ & $4 / 15$ & $7.7546 \times 10^{-4}$ & $1.3488 \times 10^{-5}$ & $1.3433 \times 10^{-5}$ & $5.4469 \times 10^{-8}$ \\
$3 / 160$ & 0.3 & $6.8350 \times 10^{-4}$ & $1.0388 \times 10^{-5}$ & $1.0323 \times 10^{-5}$ & $6.9368 \times 10^{-8}$ \\
0.02 & 0.32 & $6.2698 \times 10^{-4}$ & $8.6851 \times 10^{-6}$ & $8.6112 \times 10^{-6}$ & $7.7093 \times 10^{-8}$ \\
0.025 & 0.4 & $3.9085 \times 10^{-4}$ & $3.2351 \times 10^{-6}$ & $3.1467 \times 10^{-6}$ & $9.3072 \times 10^{-8}$ \\
$1 / 34$ & $8 / 17$ & $4.5965 \times 10^{-4}$ & $5.2537 \times 10^{-6}$ & $5.2510 \times 10^{-6}$ & $3.708 \times 10^{-9}$ \\
0.03 & 0.48 & $1.4596 \times 10^{-4}$ & $4.6945 \times 10^{-7}$ & $3.9599 \times 10^{-7}$ & $7.7350 \times 10^{-8}$ \\
0.04 & 0.64 & $1.1718 \times 10^{-3}$ & $2.4062 \times 10^{-5}$ & $2.3462 \times 10^{-5}$ & $6.0153 \times 10^{-7}$ \\
\hline
\end{tabular}




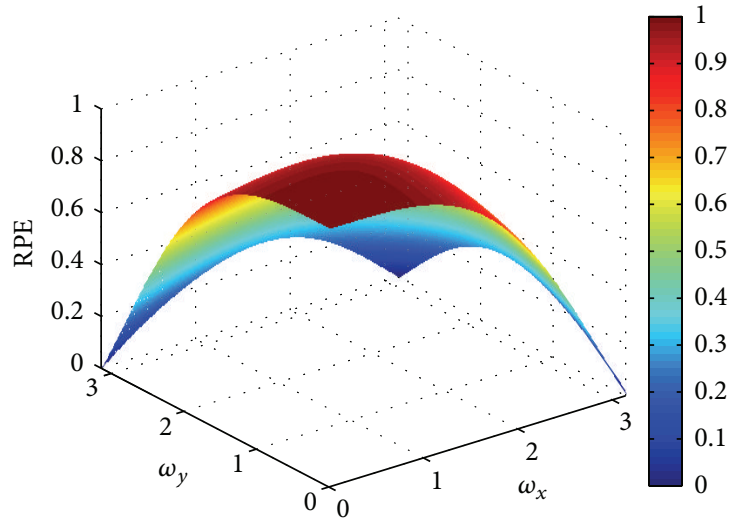

(a) $h=0.05, k=0.01$

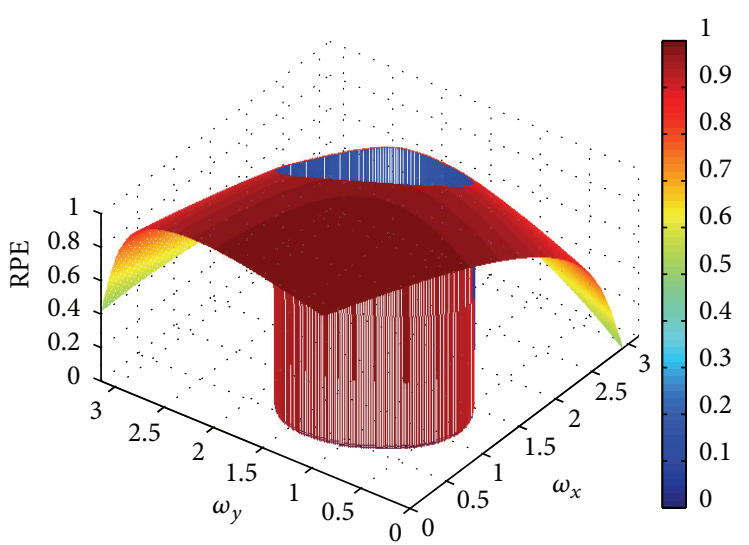

(c) $h=0.05, k=0.03$

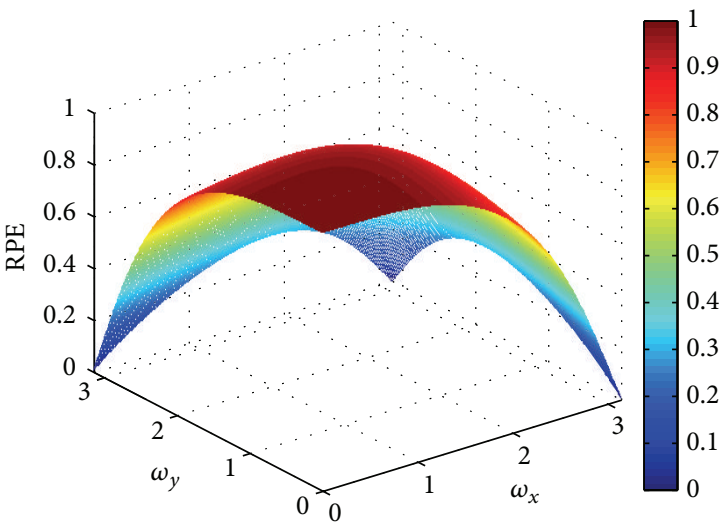

(b) $h=0.05, k=0.02$

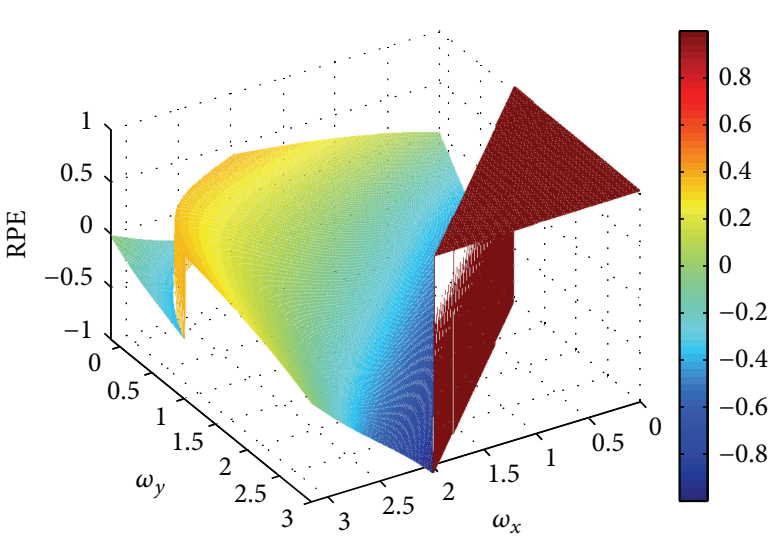

(d) $h=0.05, k=0.05$

FIGURE 11: 3D plots of relative phase error versus $\omega_{x}$ versus $\omega_{y}$ at $h=0.05$ at some different values of $k$ for the LOD $(1,5)$ procedure.

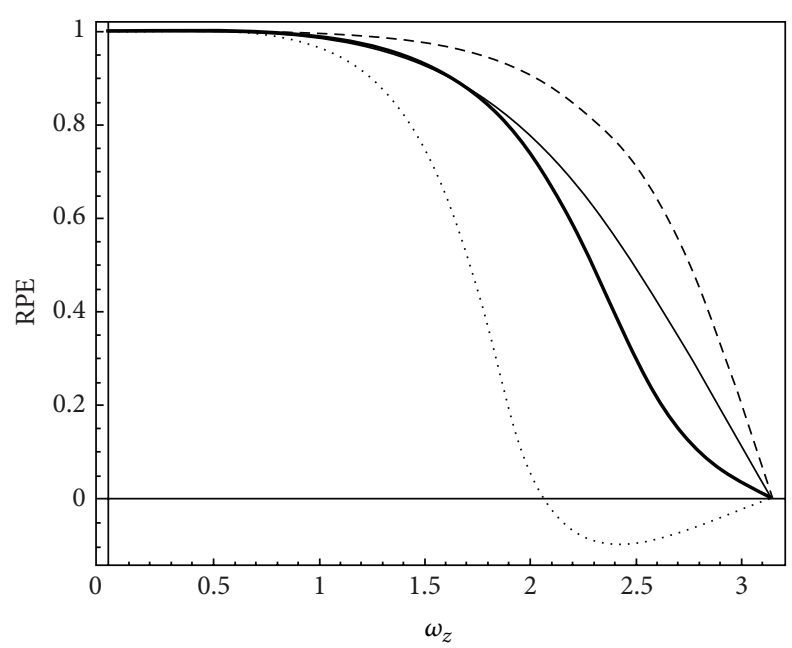

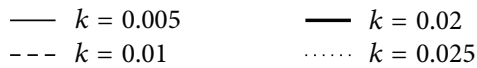

(a) $h=0.025$

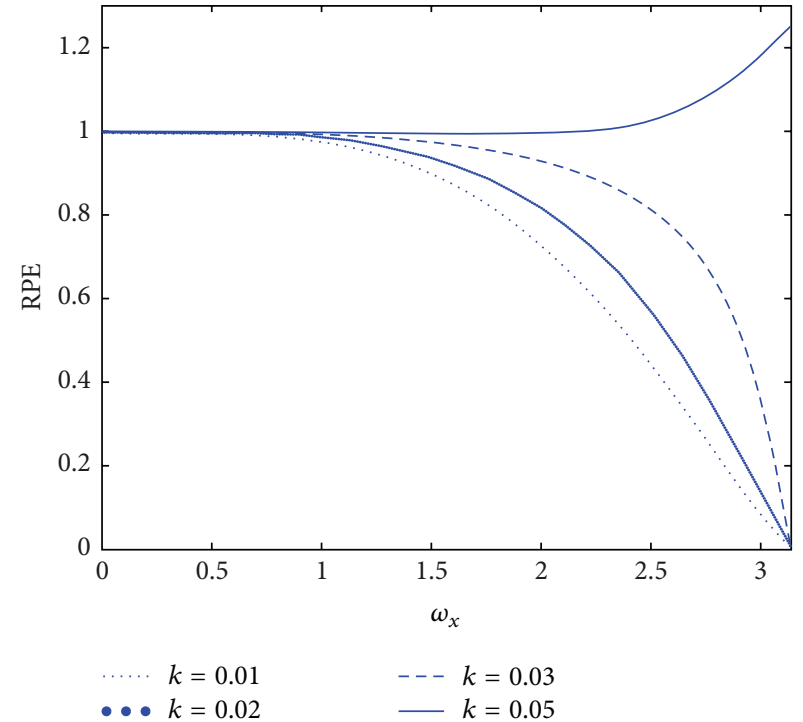

(b) $h=0.05$

FIGURE 12: Plots of relative phase error versus $\omega_{x}$ when $\omega_{y}=0$ at $h=0.025$ and $h=0.05$ with some different values of $k$ for the LOD $(1,5)$ procedure. 


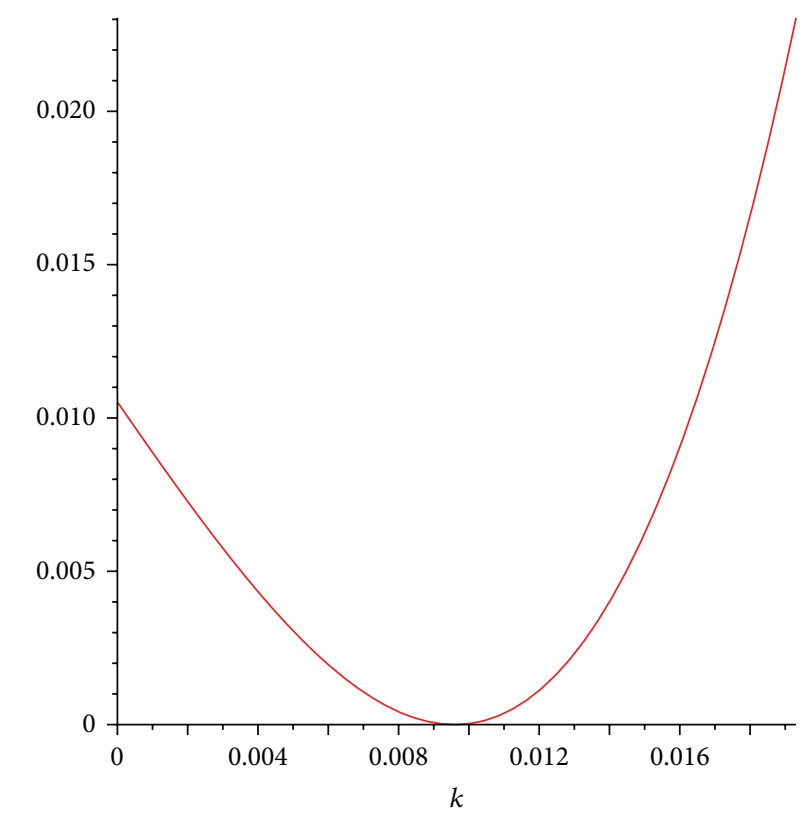

(a) LOD Lax-Wendroff

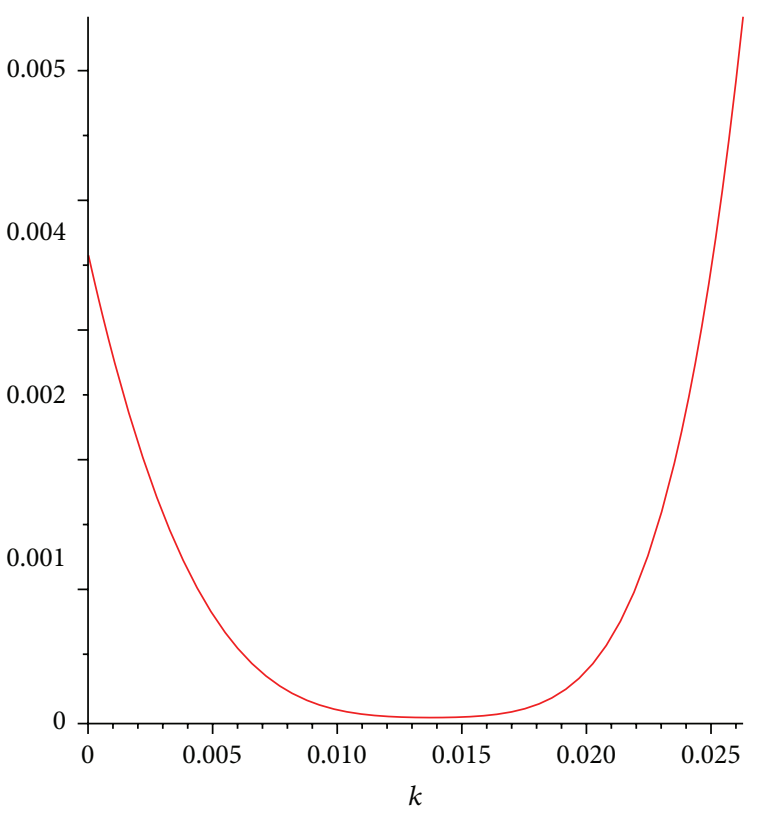

(b) $\operatorname{LOD}(1,5)$ procedure

FIGURE 13: Plots of integrated errors versus $k$ at $h=0.025$ for the two time-splitting schemes.

TABLE 3: Errors obtained from the $\operatorname{LOD}(1,5)$ procedure at $h=0.025$.

\begin{tabular}{lccccc}
\hline$k$ & CFL & $E_{\text {num }}$ & Total mean square error & DISP. ERROR & DISS. ERROR \\
\hline 0.005 & 0.16 & $4.0539 \times 10^{-4}$ & $4.1593 \times 10^{-6}$ & $4.0009 \times 10^{-6}$ & $1.6455 \times 10^{-7}$ \\
0.01 & 0.32 & $4.0072 \times 10^{-4}$ & $4.1573 \times 10^{-6}$ & $3.9874 \times 10^{-6}$ & $1.6540 \times 10^{-7}$ \\
$1 / 60$ & $8 / 15$ & $4.0565 \times 10^{-4}$ & $4.1577 \times 10^{-6}$ & $3.9797 \times 10^{-6}$ & $1.6390 \times 10^{-7}$ \\
$3 / 160$ & 0.60 & $3.9839 \times 10^{-4}$ & $4.1494 \times 10^{-6}$ & $3.9782 \times 10^{-6}$ & $1.6268 \times 10^{-7}$ \\
0.02 & 0.64 & $3.9878 \times 10^{-4}$ & $4.1481 \times 10^{-6}$ & $3.9768 \times 10^{-6}$ & $1.6174 \times 10^{-7}$ \\
0.025 & 0.80 & $4.0890 \times 10^{-4}$ & $4.1640 \times 10^{-6}$ & $4.0430 \times 10^{-6}$ & $1.5618 \times 10^{-7}$ \\
\hline
\end{tabular}

measures and consequently their technique of optimization in computational aeroacoustics.

The dispersion-relation-preserving (DRP) scheme was designed so that the dispersion relation of the finite difference scheme is formally the same as that of the original partial differential equations. The integrated error is defined as

$$
E=\int_{-\eta}^{\eta}\left|\theta^{*} h-\theta h\right|^{2} d(\theta h)
$$

where the quantities $\theta^{*} h$ and $\theta h$ represent the numerical and exact wavenumbers, respectively. The dispersion error and dissipation error are calculated as $\left|\Re\left(\theta^{*} h\right)-\theta h\right|$ and $\left|\operatorname{Im}\left(\theta^{*} h\right)\right|$, respectively.

Tam and Shen [21] set $\eta$ as 1.1 and optimize the coefficients in the numerical scheme such that the integrated error is minimized.

Bogey and Bailly minimize the relative difference between the exact wavenumber $\theta h$ and the effective/numerical wavenumber $\theta^{*} h$ and define their integrated errors as

$$
E=\int_{(\theta h)_{l}}^{(\theta h)_{h}} \frac{\left|\theta^{*} h-\theta h\right|}{\theta h} d(\theta h)
$$

or

$$
E=\int_{\ln (\theta h)_{l}}^{\ln (\theta h)_{h}}\left|\theta^{*} h-\theta h\right| d(\ln (\theta h)) .
$$

In computational fluid dynamics for a particular method under consideration, the dispersion error is calculated as

$$
|1-\mathrm{RPE}| \text {. }
$$

We have modified the measures used by Tam and Webb and Bogey and Bailly in a computational aeroacoustics framework to suit them in a computational fluid dynamics framework [22] such that the optimal parameter can be obtained. We have defined the following integrated errors integrated error from Tam and Webb (IETAM) integrated error from Bogey and Bailly (IEBOGEY) [22] as follows:

$$
\begin{gathered}
\text { IETAM }=\int_{0}^{w_{1}}|1-\mathrm{RPE}|^{2} d w, \\
\text { IEBOGEY }=\int_{0}^{w_{1}}|1-\mathrm{RPE}| d w .
\end{gathered}
$$


TABLE 4: Errors obtained from the LOD $(1,5)$ procedure at $h=0.05$.

\begin{tabular}{lccccc}
\hline$k$ & CFL & $E_{\text {num }}$ & Total mean square error & DISP. ERROR & DISS. ERROR \\
\hline 0.01 & 0.16 & $2.0000 \times 10^{-4}$ & $7.8149 \times 10^{-7}$ & $7.7377 \times 10^{-7}$ & $1.1297 \times 10^{-8}$ \\
0.02 & 0.32 & $1.3905 \times 10^{-4}$ & $3.9277 \times 10^{-7}$ & $3.8065 \times 10^{-7}$ & $1.4712 \times 10^{-8}$ \\
$1 / 34$ & $8 / 17$ & $4.1603 \times 10^{-4}$ & $4.0763 \times 10^{-6}$ & $4.0663 \times 10^{-6}$ & $1.1482 \times 10^{-8}$ \\
0.03 & 0.48 & $9.5979 \times 10^{-5}$ & $2.1598 \times 10^{-7}$ & $2.0584 \times 10^{-7}$ & $1.4193 \times 10^{-8}$ \\
0.05 & 0.8 & $7.9301 \times 10^{-5}$ & $1.7934 \times 10^{-7}$ & $1.7059 \times 10^{-7}$ & $1.0449 \times 10^{-8}$ \\
0.0625 & 1 & $8.5551 \times 10^{-4}$ & $1.4674 \times 10^{-5}$ & $1.4310 \times 10^{-5}$ & $3.6790 \times 10^{-7}$ \\
\hline
\end{tabular}

In [8], the integrated error for a scheme discretising the $1 \mathrm{D}$ advection-diffusion equation

$$
\frac{\partial u}{\partial t}+\frac{\partial u}{\partial x}=0.01 \frac{\partial^{2} u}{\partial x^{2}}
$$

is obtained as

$$
\int_{0}^{1.1}(\mathrm{RPE}-1)^{2} d \omega
$$

The value of $h$ was fixed as 0.02 and the range of values of $k$, was determined. Then the integrated error, which is a function of $k$, was minimized and the optimal value of $k$ is determined using NLPSolve function in maple.

We extend the work on optimization of parameters in [8] which is for the $1 \mathrm{D}$ advection-diffusion equation for the case of the $2 \mathrm{D}$ advection-diffusion equation. We define the integrated error as

$$
\int_{0}^{1.1} \int_{0}^{1.1}(\mathrm{RPE}-1)^{2} d \omega_{x} d \omega_{y} .
$$

We first obtain an expression for the RPE of the LOD LaxWendroff when discretizing the equation

$$
\frac{\partial u}{\partial t}+0.8 \frac{\partial u}{\partial x}+0.8 \frac{\partial u}{\partial y}=0.01 \frac{\partial^{2} u}{\partial x^{2}}+0.01 \frac{\partial^{2} u}{\partial y^{2}}
$$

Since $c=0.8 k / h$ and $s=0.8 k / h^{2}$ and we choose $h=$ 0.025 , we have $c=32 k$ and $s=16 k$. Hence, the RPE is a function of $k, \omega_{x}$, and $\omega_{y}$. Since we can have phase wrapping, we make use of Taylor's expansion to obtain an approximation for the RPE up to the terms

$$
\left(\omega_{x}\right)^{3}\left(\omega_{y}\right)^{2},\left(\omega_{x}\right)^{5},\left(\omega_{x}\right)^{2}\left(\omega_{y}\right)^{3},\left(\omega_{y}\right)^{5}, \omega_{x}\left(\omega_{y}\right)^{4},\left(\omega_{x}\right)^{4} \omega_{y} .
$$

The integrated error $\int_{0}^{1.1} \int_{0}^{1.1}(\mathrm{RPE}-1)^{2} d \omega_{x} d \omega_{y}$ is obtained by using Simpson's method and it is a function of $k$ only. A plot of the integrated error versus $k$ is shown in Figure 13(a) for $k \in[0,0.0193]$. Using the NLPSolve function in maple, this optimal value of $k$ is found to be 0.009593 and the minimum value of the integrated error is $1.883960 \times 10^{-7}$.

To validate our results, we perform the same numerical experiment described in Section 3.2 at $h=0.025$ and use the optimal value of $k$ or a value of $k$ close to this optimal value, in that case, $k=3 / 310 \approx 0.0096$, and compute the errors. The error rate, total mean square error, and dispersion error are $3.9967 \times 10^{-4}, 4.1603 \times 10^{-6}, 3.9878 \times 10^{-6}$. These three errors are all least as compared to when other values of $k$ are used as shown in Table 1.

We adopt the same procedure to compute the optimal value of $k$ for the $\operatorname{LOD}(1,5)$ scheme when $h=0.025$. We obtain an approximate expression for the RPE of the LOD $(1,5)$ when $c=32 k$ and $s=16 k$. We use Simpson's rule to approximate the integral given by (90) which is a function of $k$. A plot of the integrated error versus $k$ for $k \epsilon$ $[0,0.026288]$ is shown in Figure 13(b) and using NLPSolve function in maple the optimal value of $k$ is 0.013782 and also the minimum value of the integral is $1.139313 \times 10^{-6}$.

We perform the numerical experiment described in Section 3.2 with $k=3 / 220$ which is close to the optimal value of $k$ we have obtained with $h=0.025$. The error rate, total mean square error, dispersion error, and dissipation error are $3.9948 \times 10^{-4}, 4.1504 \times 10^{-6}, 3.9459 \times 10^{-6}$, and $1.6501 \times 10^{-7}$, respectively. The total mean square error and dispersion error are both least when $k=3 / 220 \approx 0.01378$.

\section{Conclusion}

In this paper, two time-splitting procedures are used to solve a $2 \mathrm{D}$ advection-diffusion equation with constant coefficients when the advection velocity in both $x$ - and $y$-directions is 0.8 and also when the coefficient of diffusivity in both $x$ and $y$-directions is 0.01 . We perform a stability analysis and spectral analysis of the dispersion and dissipation properties of the two schemes at some values of $h$ and $k$. Numerical experiments are carried out and various errors are computed. These errors are dependent on the values of $h$ and $k$. It is observed that in general the dispersion error is more affected by the values of $k$ and $h$ for the LOD Lax-Wendroff scheme as compared to that of the $\operatorname{LOD}(1,5)$ scheme at a given value of $h$. We then use an optimization technique based on minimisation of the square of the dispersion error to find the optimal value of $k$ when $h$ is chosen as 0.025 and this is validated by numerical experiments.

Future extension of this work to consider other types of advection-diffusion equations when dissipation dominates and to find out which optimization techniques are suitable in these cases. Also, the work can be extended to 2D nonlinear convection-diffusion problems. 


\section{Nomenclature}

$\begin{array}{ll}I: & \sqrt{(-1)} \\ h: & \text { Spatial step } \\ k: & \text { Time step } \\ R_{\Delta}: & \text { Reynolds number } \\ \text { RPE: } & \text { Relative phase error per unit time step } \\ \text { AFM: } & \text { Modulus of amplification factor } \\ \beta_{x}: & \text { Advection velocity in } x \text {-direction } \\ \beta_{y}: & \text { Advection velocity in } y \text {-direction } \\ \beta_{z}: & \text { Advection velocity in } z \text {-direction } \\ \alpha_{x}: & \text { Coefficient of diffusivity in } x \text {-direction } \\ \alpha_{y}: & \text { Coefficient of diffusivity in } y \text {-direction } \\ \alpha_{z}: & \text { Coefficient of diffusivity in } z \text {-direction } \\ \theta_{1}: & \text { Wavenumber in } x \text {-direction } \\ \theta_{2}: & \text { Wavenumber in } y \text {-direction } \\ \omega_{x}: & \text { Phase angle in } x \text {-direction } \\ \omega_{y}: & \text { Phase angle in } y \text {-direction } \\ \omega_{x}: & \Delta x \theta_{1} \\ \omega_{y}: & \Delta y \theta_{2} \\ c_{x}: & \beta_{x} k / h \\ c_{y}: & \beta_{y} k / h \\ s_{x}: & \alpha_{x} k / h^{2} \\ s_{y}: & \alpha_{y} k / h^{2} \\ \text { DISP. ERROR: } & \text { Dispersion error } \\ \text { DISS. ERROR: } & \text { Dissipation error. } \\ & \end{array}$

\section{Acknowledgments}

The work of Dr A. R. Appadu was funded through the Research Development Programme of the University of Pretoria and the period of funding is from January 2013 to January 2014. Mr Hagos is grateful to the African Institute for Mathematical Sciences (AIMS), South Africa, for a full bursary for the structured Master's program from August 2012 to July 2013. The authors are grateful to the two anonymous reviewers for their comments which were useful in clarifying and focusing the presentation.

\section{References}

[1] A. Shukla, A. K. Singh, and P. Singh, "A recent development of numerical methods for solving convection-diffusion problems," Applied Mathematics, vol. 1, pp. 1-12, 2011.

[2] M. Dehghan, "On the numerical solution of the one-dimensional convection-diffusion equation," Mathematical Problems in Engineering, vol. 2005, no. 1, pp. 61-74, 2005.

[3] M. Dehghan, "Time-splitting procedures for the solution of the two-dimensional transport equation," Kybernetes, vol. 36, no. 56, pp. 791-805, 2007.

[4] C. B. Laney, Computational Gasdynamics, Cambridge University Press, Cambridge, UK, 1998.

[5] J. E. Fromm, "A method for reducing dispersion in convective difference schemes," Journal of Computational Physics, vol. 3, no. 2, pp. 176-189, 1968.

[6] R. J. Babarsky and R. Sharpley, "Expanded stability through higher temporal accuracy for time-centered advection schemes," Monthly Weather Review, vol. 125, no. 6, pp. 1277-1295, 1997.
[7] K. W. Morton and D. F. Mayers, Numerical Solution of Partial Differential Equations, Cambridge University Press, Cambridge, UK, 1994.

[8] A. R. Appadu, "Numerical solution of the 1D advection-diffusion equation using standard and nonstandard finite difference schemes," Journal of Applied Mathematics, vol. 2013, Article ID 734374, 14 pages, 2013.

[9] R. Smith and Y. Tang, "Optimal and near-optimal advectiondiffusion finite-difference schemes. V. Error propagation," Proceedings of the Royal Society of London A, vol. 457, no. 2008, pp. 803-816, 2001.

[10] C. Hirsch, Numerical Computation of Internal and External Flows, vol. 1, John Wiley \& Sons, New York, NY, USA, 1988.

[11] R. W. MacCormack and A. J. Paullay, "Computational efficiency achieved by time splitting of finite difference operators," Tech. Rep. AIAA Paper 72-154, 1972.

[12] M. Dehghan, "Weighted finite difference techniques for the one-dimensional advection-diffusion equation," Applied Mathematics and Computation, vol. 147, no. 2, pp. 307-319, 2004.

[13] L. L. Takacs, "A two-step scheme for the advection equation with minimized dissipation and dispersion errors," Monthly Weather Review, vol. 113, no. 6, pp. 1050-1065, 1985.

[14] A. R. Appadu and M. Z. Dauhoo, “The concept of minimized integrated exponential error for low dispersion and low dissipation schemes," International Journal for Numerical Methods in Fluids, vol. 65, no. 5, pp. 578-601, 2011.

[15] A. R. Appadu, "Some applications of the concept of minimized integrated exponential error for low dispersion and low dissipation," International Journal for Numerical Methods in Fluids, vol. 68, no. 2, pp. 244-268, 2012.

[16] A. C. Hindmarsh, P. M. Gresho, and D. F. Griffiths, "The stability of explicit Euler time-integration for certain finite difference approximations of the multi-dimensional advection-diffusion equation," International Journal for Numerical Methods in Fluids, vol. 4, no. 9, pp. 853-897, 1984.

[17] E. Sousa, "The controversial stability analysis," Applied Mathematics and Computation, vol. 145, no. 2-3, pp. 777-794, 2003.

[18] C. K. W. Tam and J. C. Webb, "Dispersion-relation-preserving finite difference schemes for computational acoustics," Journal of Computational Physics, vol. 107, no. 2, pp. 262-281, 1993.

[19] C. Bogey and C. Bailly, "A family of low dispersive and low dissipative explicit schemes for flow and noise computations," Journal of Computational Physics, vol. 194, no. 1, pp. 194-214, 2004.

[20] R. Hixon, "On increasing the accuracy of MacCormack schemes for aeroacoustic applications," Tech. Rep. AIAA Paper 10.2514/6.1997-1586, 1997.

[21] C. K. W. Tam and H. Shen, "Direct computation of nonlinear acoustic pulses using high-order finite differences schemes," Tech. Rep. AIAA Paper 93-4325, 1993.

[22] A. R. Appadu, "Comparison of some optimisation techniques for numerical schemes discretising equations with advection terms," International Journal of Innovative Computing and Applications, vol. 4, no. 1, pp. 12-27, 2012. 


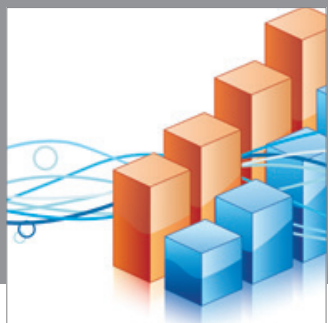

Advances in

Operations Research

mansans

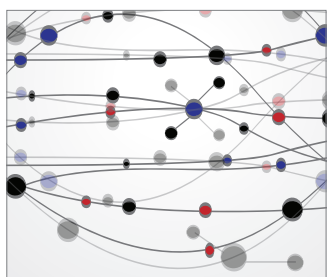

The Scientific World Journal
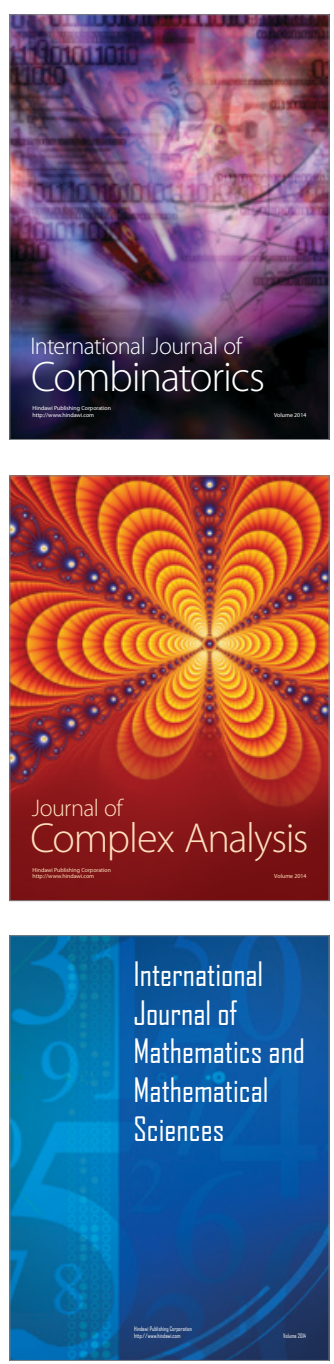
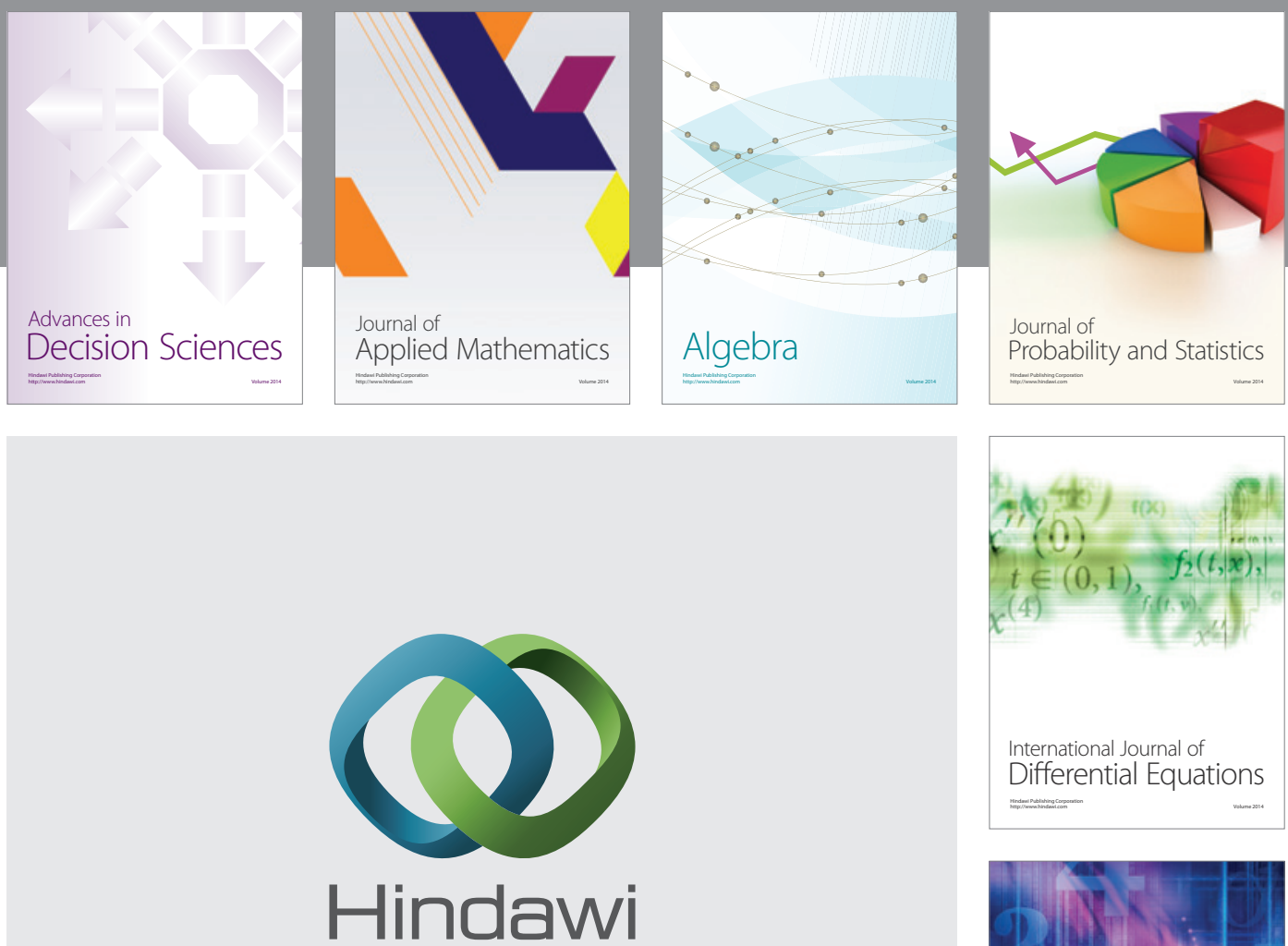

Submit your manuscripts at http://www.hindawi.com
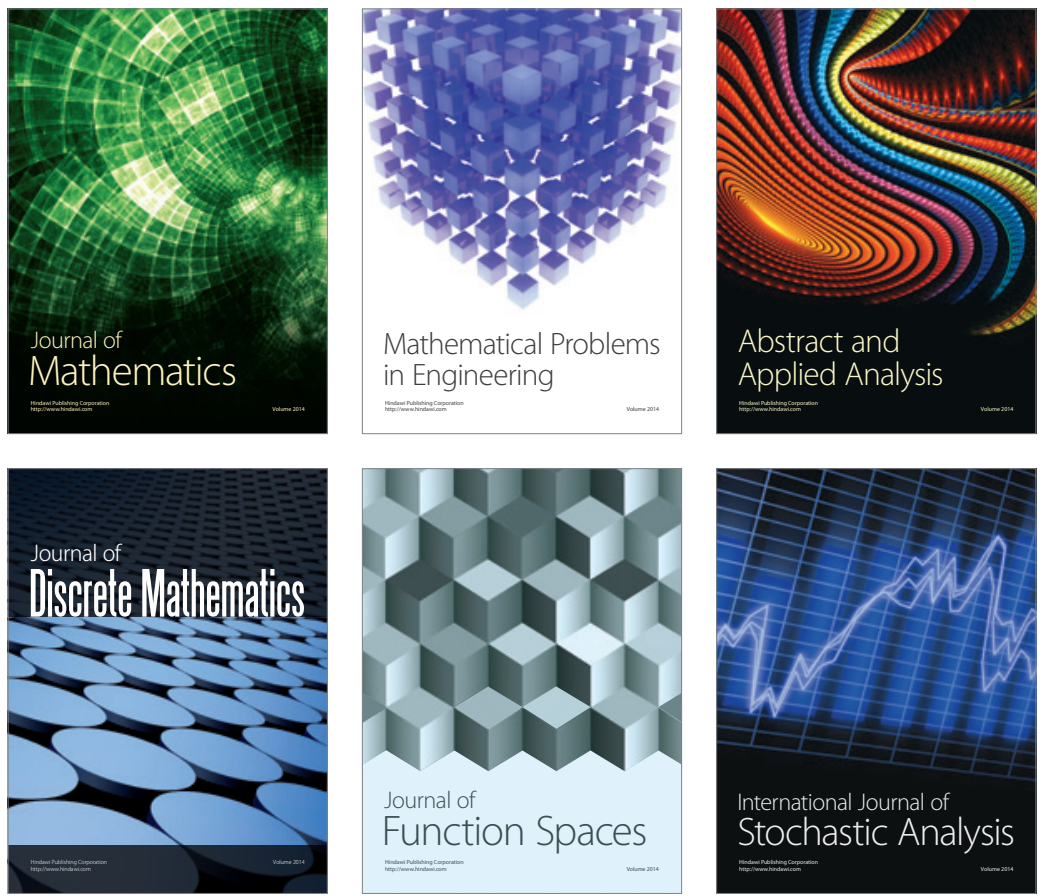

Journal of

Function Spaces

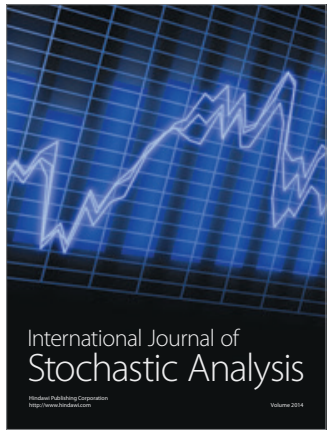

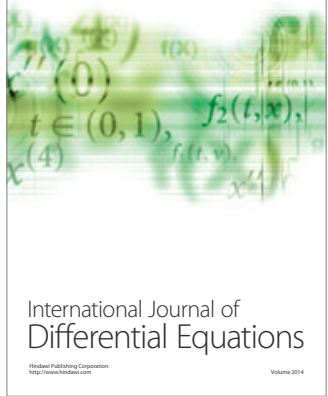
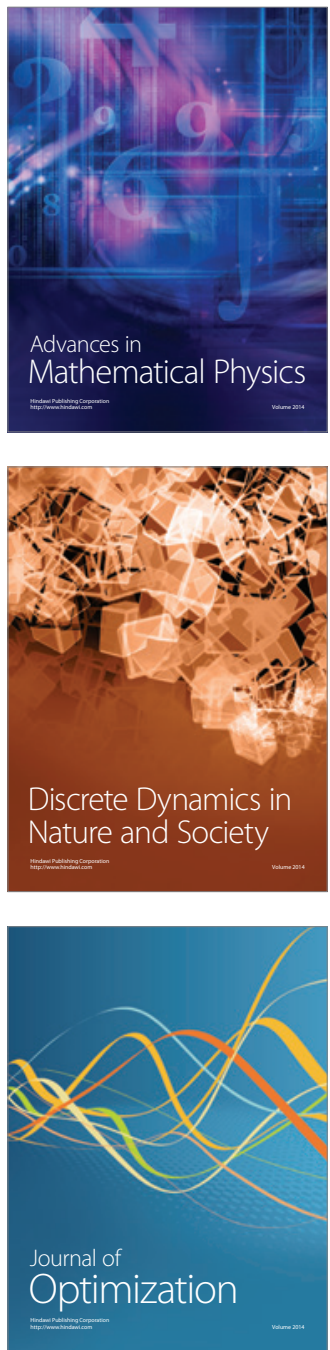